\title{
Volume of Trade and Dynamic Network Formation in Two-Sided Economies $^{1}$
}

\section{Roland Pongou ${ }^{2}$ and Roberto Serrano ${ }^{3}$}

This version: June 2013

\begin{abstract}
We study the long-run stability of trade networks in a two-sided economy of agents labelled men and women. Each agent desires relationships with the other type, but having multiple partners is costly. This cost-benefit trade-off results in each agent having a single-peaked utility over the number of partners-the volume of trade-, the peak being greater for men than for women. We propose a stochastic matching process in which self-interested agents form and sever links over time. Links can be added or deleted, sometimes simultaneously by a single agent. While the unperturbed process yields each pairwise stable network as an absorbing state, stochastic stability in two perturbed processes provides a significant refinement, leading respectively to egalitarian and anti-egalitarian pairwise stable networks. This has implications for the concentration on each side of the market of a random information shock. The analysis captures stylized facts, related to market fragmentation, concentration and contagion asymmetry, in several two-sided economies.
\end{abstract}

JEL classification numbers: A14, C7, I12, J00

Keywords: Two-sided networks, pairwise stability, stochastic stability, herd externality, informational cascade, fragmentation, concentration, economy thinness, contagion asymmetry.

\footnotetext{
${ }^{1}$ We are grateful to Victoria Barham, Francis Bloch, Max Blouin, Antonio Cabrales, Pedro Dal Bó, Geoffroy de Clippel, Mark Dean, Kfir Eliaz, Marcel Fafchamps, Andrew Foster, Oded Galor, Elhanan Helpman, Matt Jackson, Larry Samuelson, Fernando Vega-Redondo, Eric Verhoogen, David Weil, and Peyton Young for comments and encouragement. Pongou gratefully acknowledges the hospitality of the CSAE at the Department of Economics of the University of Oxford. Serrano gratefully acknowledges research support from Spain's Ministry of Science and Innovation under grant Consolider 2010 CSD2006-0016 and thanks CEMFI for its hospitality.

${ }^{2}$ Dept. of Economics, University of Ottawa, Ontario, Canada; rpongou@uottawa.ca

${ }^{3}$ Dept. of Economics, Brown University, Providence, RI 02912, U.S.A.; roberto_serrano@brown.edu
} 


\section{Introduction}

We propose a dynamic theory of network formation in a two-sided economy. Although the model covers several applications, where the sides of the market could be rich/poor countries, buyers/sellers, employers/workers, faculty/students, and so on, to fix ideas, we shall refer to agents on both sides as men and women, respectively, and we shall assume that both are of equal size. Each agent derives utility from having relationships with the other type. However, having many partners is costly. This cost-benefit trade-off results in each agent having a single-peaked utility function. Preference heterogeneity is possible, but we assume peak-homogeneity within each side of the economy. Moreover, our key maintained assumption is that the peak (or optimal number of partners) is greater for men than for women (i.e., one side of the market wishes to have more links with the other side).

A link between a man and a woman might be viewed as representing a trade relationship in which the two parties exchange "goods" of some sort. A good might be material like in a real exchange market, or emotional such as among lovers. Agents derive utility only from the number of partners they have, which we call the volume of trade. Although each relationship involves an exchange of goods, we choose to model network formation as in a repugnant market (Roth (2007)) where links cannot be sold or bought.

Our goal is two-fold. First, building on the study of the static stability of networks, we extend the analysis to a dynamic setting, yielding a characterization of networks that arise in the very long run. This characterization provides a rationale behind certain phenomena of market fragmentation and concentration in several two-sided economies. Second, we apply a new index of communication and contagion to these long-run networks to study asymmetry in the concentration of a random information shock. ${ }^{1}$

The current analysis contributes to two broad literatures: the literature on two-sided matching (with capacity constraints), and the literature on endogenous network formation. Its distinctive feature, however, is that agents in our model do not direct their links but decide the number of partners (or the volume of trade). Here, the link formation process is not equivalent to elaborating a nominal list of intended relationships, as is the case in these literatures. ${ }^{2}$ This simplification enables us to totally characterize statically and long-run stable matchings in terms of the allocation of links between partners and to study their diffusion properties for each side of the economy.

Our model may be used in a wide variety of contexts where agents mostly care about the number of partners they have. Fidelity economies are an example (Pongou (2010)). In these economies, having many partners of the opposite type may be viewed as infidelity, which is punished if detected. An example of such a market is

\footnotetext{
${ }^{1}$ The nature of the shock depends on the type of the economy. For instance, in a faculty-student economy, a shock might be a new idea or research question. In a dating economy, a shock might be a new sex technology or a sexually transmitted disease.

${ }^{2}$ Although agents might differ in various characteristics in a two-sided market with buyers and sellers, for instance, we assume that these characteristics do not enter their potential partners' utility functions. A chocolate seller might not care about whether his buyers are tall or short, white, black, or purple: he only cares about the number of sales.
} 
an employer-employee market where a contract stipulates a prima facie duty of loyalty of an employee to her employer, which precludes working for a competing firm. Here, the requirement of fidelity is only one-sided. Another example of a fidelity economy is the dating market (Pongou and Serrano (2013)).

The model has several other applications, including buyers and sellers transacting in a market for a continuous good. In this case, one can argue that the optimal number of buyers for each seller exceeds the optimal number of stores each buyer purchases from. Another example is the international market between rich and poor countries, where the optimal number of trading partners is generally greater for the former than for the latter. Yet another example is the economy involving graduate students writing a doctoral dissertation and their faculty advisors. Here, the number of optimal links for students (the size of the doctoral committee) is usually lower than it is for professors (the number of committees they can join). The instructor-student market in a department of Economics is another application of our model. Here too, each instructor may have several students while each student may only take a small number of courses from different instructors.

In what follows, we provide an overview of the findings, shedding light on the patterns of relationships that form in two-sided economies such as the ones just mentioned, and showing how these relationships subsequently affect the spread of information, with likely different outcomes for each side.

\subsection{Static Analysis of Two-Sided Networks: Pairwise Stability}

We first characterize the pairwise stable networks. In a matching problem such as ours, individuals form new links or sever existing ones based on the reward that the resulting network offers them relative to the current network. For simplicity, as in most of the literature, agents are myopic. We say that a network is pairwise stable if: (i) no individual has an incentive to sever an existing link he or she is involved in, and (ii) no pair of a man and a woman have a strict incentive to form a new link between them while at the same time possibly severing some of the existing links they are involved in. ${ }^{3}$

We shall make an assumption, met when populations are sufficiently large, which allows for a simple characterization of pairwise stable networks. ${ }^{4}$ In particular, we find that a network is pairwise stable if and only if each woman has exactly her optimal number of partners, and each man has at most his optimal number of partners. Women supply a smaller number of links than the ones demanded by men, which in turn results in only men competing for female partners while each woman is sure of having the number of male partners she desires.

\footnotetext{
${ }^{3}$ See Gale and Shapley (1962) for a first use of pairwise stability. Within networks, Jackson and Wolinsky (1996) provide the standard definition. Our definition is slightly different: while they allow weak blocking in the pair, we assume strict blocking, in part due to the absence of side-payments.

${ }^{4}$ Pongou (2010, Chapter 2) provides a full characterization of pairwise stable networks without the assumption made here, but unlike the current paper, his analysis is only static.
} 


\subsection{Unperturbed Dynamic Analysis: Steady-State Networks}

The center of our analysis is a dynamic matching process for the matching problem, more precisely a Markov process. ${ }^{5}$ Random encounters between men and women are based on the incentives that agents have to form new links or sever existing ones. Specifically, the unperturbed Markov process assumes discrete time, and is defined as follows. In each period, a man and a woman chosen at random with arbitrary positive probability are given the opportunity to sever or add a link based on the improvement that the resulting network offers to each of them relative to the current network. If they are already linked in the current network, the decision is whether to sever the link; severance is a unilateral decision. Otherwise, the decision is whether to form a new link; link formation is a bilateral decision. While forming a new link, each agent is allowed to sever as many of the links he/she is involved in as possible in the current network (although, because of our single-peaked preferences assumption, without loss of generality, one can restrict attention to the case of severing only one link). The long-run predictions -steady or recurrent states- of this process coincide with the set of pairwise stable networks, a very large set. Such a large set does not deliver any clear result in terms of contagion asymmetry across the two sides of the economy.

\subsection{Perturbed Dynamic Analysis: Stochastically Stable Networks}

To gain predictive power in our analysis, the matching process is perturbed in two different ways, corresponding to two different perceptions of multiple partnerships. Each perturbation consists of allowing a small probability of forming new links or severing existing ones when this action is not beneficial to the agents involved. We study the long-run predictions of these perturbed processes -their stochastically stable networks-, these predictions being the only networks that are visited a positive proportion of time in the very long run. ${ }^{6}$

In both perturbed dynamic processes, if a link formation is mutually beneficial or if a link severance is beneficial to its initiator, it occurs with probability 1 . That is, this feature of the unperturbed dynamics is retained. However, the perturbed processes allow for more transitions. As it turns out, using uniform perturbations (e.g., as in Jackson and Watts (2002)), does not serve as a useful refinement in our model. Thus, we are led to explore other possibilities, which we motivate with extra-economic elements of the society. Specifically, in both processes, an action that worsens its initiator, which we shall call a mistake, occurs with a small probability $\varepsilon>0$. But key to our analysis are actions that are neither beneficial nor mistakes, i.e., actions that leave their initiators exactly indifferent. We shall refer to these as utility neutral actions or neutral

\footnotetext{
${ }^{5}$ Jackson and watts (2002) also provide a related analysis of dynamic networks. We compare our results to theirs and others in the literature in the sequel.

${ }^{6}$ In a perturbed process, one can no longer speak of "steady states," as by definition, there is always a positive probability of transiting from any state to any other. The notion of stochastic stability (Freidlin and Wentzell (1984)) provides a useful methodology to identify those states in which the perturbed process spends most of its time in the long run. It has been applied to study a number of problems in the economics literature (see, e.g., Foster and Young (1990), Kandori, Mailath and Rob (1993), Young (1993) for early contributions). Young (1998) presents many of its applications. The main shortcoming of stochastic stability is its associated slow speed of convergence, but it is very helpful in identifying long-run trends, our main interest here. Also, the reader should keep in mind that the frequency of a random encounter between a man and a woman may be extremely high, perhaps every minute depending on the type of market being considered, thus allaying the issue.
} 
actions for short. In the spirit of assuming that more serious mistakes are less likely, an agent's probability of taking a neutral action will always exceed $\varepsilon$. We explain how.

In our models, neutral actions uniquely correspond to situations in which an agent severs an existing link with a current partner and forms a new link with another agent. We shall assume that the probability of taking such a neutral action is $\varepsilon^{f(\cdot)}$ (a number strictly greater than $\varepsilon$ because the exponent will be a number between 0 and 1). The exponent is the "perceived" strength of the existing link so that, in evaluating the merits of taking a neutral action, links that are perceived as stronger $-f(\cdot)$ closer to $1-$ are harder to break.

In the first perturbed process, the strength $f(\cdot)$ of a link to be severed is inversely proportional to the number of partners that the partner to be abandoned has in the existing network. One interpretation is that this link is perceived as strong as the amount of time invested in it by that partner. In this process, we find that networks are stochastically stable if and only if they are egalitarian pairwise stable networks. Men and women have the same number of partners, which is the optimal number of partners for women.

In contrast, the second perturbed process assumes that the strength $f(\cdot)$ of a link to be severed is directly proportional to the number of partners that the partner to be left behind has in the existing network. There are several interpretations of this process. The individual who invests more time in a relationship either shows relative weakness to his/her partner, or (perhaps wrongly) signals the "high quality" of the latter to other agents. $^{7}$ For this case, we find that anti-egalitarian pairwise stable networks, which are networks in which each woman has her optimal number of partners, and the smallest possible set of men is matched, will be the only ones visited a positive proportion of time in the very long run. Each non-isolated man is matched to his optimal number of partners (except for at most one matched man, who will be matched to the remaining women). The rest of men will remain isolated.

Each of the two stochastic processes may be interpreted differently depending on the context to which it applies. In a fidelity economy such as a dating market, for instance, the first process might be viewed as describing a situation in which the amount of time invested in a relationship seems to signal commitment. A perceived stronger commitment of one partner in a relationship makes it harder to break by the other partner. A salient particular outcome of this process when the optimal number of partners for women is 1 is the sociological phenomenon known as serial monogamy, which is associated with high divorce rates and is more common in Western societies. The second process, in contrast, might be interpreted as depicting a situation where the amount of time invested in a relationship might be evidence of submissiveness to the other partner. Investing more time therefore means being weaker; and thus, it is easier for the dominant partner to break the relationship. ${ }^{8}$ In the special case where each woman optimally has one partner, polygyny (polygamy

\footnotetext{
${ }^{7}$ For instance, a man who receives too much attention from many women might be wrongly perceived by other women as being of higher quality than one who receives less attention.

${ }^{8}$ For a possible justification of the assumption in this second process in a sexual economy, see Tertilt (2005) and further evidence from anthropologists (Pat Caldwell (1976), John C. Caldwell (1976), John C. Caldwell, Pat Caldwell and Orubuloye (1992), Quale (1992)). Some of these studies highlight the dominant role of men in male-female relationships as measured, for instance, by the small amount of time that men spend with their wives in societies like sub-Saharan Africa (John C. Caldwell $(1976)$ ), which markedly contrasts with what is observed in the West. Our assumption differs in that it is gender-neutral: in a
} 
involving several women matched to one man) is selected.

We shall later apply the two processes to several real-life economies, providing a rationale for patterns of market fragmentation and concentration that are generally observed. There are several advantages to considering both perturbed processes. ${ }^{9}$ Theoretically, studying polar opposites in the assumptions behind neutral actions offers a more complete understanding of the problem, and the rationale behind these actions offers an interesting alternative to justify the perturbations (in addition to mutations, experimentation or mistakes, invoked in previous literature). Empirically, the two approaches are consistent with different perceptions or sociological realities, prevalent in different societies or market cultures.

\subsection{Contagion Asymmetry}

In a two-sided economy, the question of which side of the economy is more likely to be more affected by the spread of a random unanticipated information shock is important. In a market involving instructors and students, for instance, the question of whether a new idea being exchanged between the two sides ends up affecting more students than instructors is important for the design of such markets. In a dating economy, one can think about the question of gender differences in the knowledge or use of a new sex technology. Also, gender inequality in the prevalence of sexually transmitted diseases is an important public health issue. In a financial market involving lenders and borrowers, it is important to determine how the spread of a financial shock affects the two sides of the market differently. In order to answer this question, we consider a simple index of contagion. Assume that an agent is drawn at random from a network to receive a piece of information. He/she then communicates it to his/her partners, who in turn communicate it to their other partners, and so on. If that agent has no partner, the information does not spread. Under the assumption that each agent is drawn with equal probability, one can define the communication or contagion potential of that network, which is the expected proportion of agents who will receive the information, and provide a formula for this notion.

One can also derive a formula for the male-female difference in contagion potential in a network. The key in these formulae is that the contagion potential in a side is proportional to the sum of squares of the agents of that side in each component of the network; see Section 8 for details.

We show that under the first perturbed dynamic process, the difference in contagion potential between the two sides of an economy in any of the stochastically stable networks is zero. Under the second process, women's contagion potential is greater than men's. ${ }^{10}$ We also find that information prevalence might differ across two

relationship between a man and a woman, the dominated partner, regardless of his/her gender, is the partner who invests more time in it. However, we show that even this more general assumption leads to networks in which connected men dominate their female partners and invest less time in their relationships.

${ }^{9}$ Bergin and Lipman (1996) show that one can always construct processes with state-dependent perturbations that will select any subset of the steady states as stochastically stable. An important implication of this result is that one should motivate the particular perturbed processes that one chooses to work with.

${ }^{10}$ This result implies that women in a heterosexual economy, and students in an instructor-student economy are more affected by the spread of a new information than the other side. This result for the stochastically stable networks of the second process may seem surprising, given that the definition of the perturbed process itself is "gender neutral". However, in combination with our assumption of asymmetry in optimal numbers of partners across agents on both sides, all the key transitions involve a woman severing a link to form a new one, and in doing so, the cost of breaking that link is a direct function of the dominant role of her former male partner, measured by the number of his links. 
identical economies. In fact, there are multiple equilibrium networks under each of the two stochastic processes we study. Therefore, the equilibrium networks that "realize" in two separate but identical economies might have different configurations, leading to the spread of a random piece of information affecting more agents in one economy than in the other.

\subsection{Plan of the Paper}

The remaining of this paper unfolds as follows. Section 2 introduces the model that forms the basis for our analysis. We characterize pairwise stable networks in Section 3. In Section 4, we define the unperturbed Markov process and characterize its recurrent or steady states. This process is perturbed in Section 5 and Section 6, and a characterization result of stochastically stable networks is provided for each of the two perturbed systems, respectively. In Section 7 we comment on the uniform perturbation model. In Section 8, we study the implications of our results for contagion asymmetry across agents types and across economies. We examine some applications in Section 9. Section 10 situates our study in the literature, and Section 11 concludes. Section 12 collects all the proofs.

\section{The Model}

The economy consists of a finite set of individuals $N=\{1,2, \ldots, n\}$, partitioned into two sets of agents labelled men $(M)$ and women $(W)$, respectively, each of equal size. Each individual derives utility from direct links with opposite type agents. Engaging in multiple links is costly. As we will see, this trade-off results in each agent having a single-peaked utility function.

\subsection{Utility Functions}

Let $\bar{M}=M \cup\{\emptyset\}$ be the expanded set of men, and $\bar{W}=W \cup\{\emptyset\}$ the expanded set of women. A network $g$ is a subset of $\bar{M} \times \bar{W}$, where $(m, \emptyset) \in g$ means that man $m$ is isolated or has no connection in $g$, and similarly, $(\emptyset, w) \in g$ means that woman $w$ has no connection in $g .{ }^{11}$

Let $g$ be a network. Since we are dealing with undirected graphs, if $(i, j) \in g$, we will abuse notation and consider that $(j, i) \in g$ (in fact, $(i, j)$ and $(j, i)$ represent the same relationship). Let $i \in N$ be an individual, and $s_{i}(g)$ the number of opposite type partners that $i$ has in the network $g$. The utility that $i$ derives from $g$ is expressed by the following function:

$$
u_{i}\left(s_{i}(g)\right)=v_{i}\left(s_{i}(g)\right)-c_{i}\left(s_{i}(g)\right)
$$

where $v_{i}\left(s_{i}(g)\right)$ is the utility derived from direct links with opposite type partners in $g$, and is concave and strictly increasing in $s_{i}(g)$; and $c_{i}\left(s_{i}(g)\right)$ the cost associated with having $s_{i}(g)$ partners, assumed to be

\footnotetext{
${ }^{11}$ More formally, for any man $m \in M$ such that $(m, \emptyset) \in g,(m, w) \notin g$ for all woman $w \in W$; similarly, for any woman $w \in W$ such that $(\emptyset, w) \in g,(m, w) \notin g$ for all man $m \in M$.
} 
increasing and convex. We also assume $v(0)=c_{i}(0)=0$, which means that having no partner brings no benefit and induces no cost.

Denote the extension of $u_{i}$ to the non-negative reals as $\overline{u_{i}}$. Without loss of generality, let $\overline{u_{i}}$ be twice continuously differentiable. The following remark is straightforward:

Remark 1 There exists $s_{i}^{*}>0$ such that ${\overline{u_{i}}}^{\prime}\left(s^{*}\right)=0$; and for any $s \in\left[0, s_{i}^{*}\right),{\overline{u_{i}}}^{\prime}(s)>0$; and for any $s \in\left(s_{i}^{*},+\infty\right),{\overline{u_{i}}}^{\prime}(s)<0$.

Remark 1 implies that each agent $i$ has a single-peaked utility function. For simplicity, we assume peakhomogeneity within each side of the economy, although agents might have "different" utility functions. Let $s_{m}^{*}$ and $s_{w}^{*}$ denote the peaks or optimal numbers of partners for each man and each woman, respectively. These numbers are natural numbers, and are found by comparing the value of the utility function in the integers immediately below and above the point of zero derivative.

In order to derive our results, we will make an assumption that implies two ideas. First, our key maintained assumption that women's desire for partners is lower than men's, and second, it is also low enough relative to the size of the population. The latter is what we refer to as a "large populations" assumption, although our model also applies to other settings. Specifically:

Assumption A1. We assume:

$$
1>\frac{s_{w}^{*}-1}{|M|}+\frac{s_{w}^{*}}{s_{m}^{*}}
$$

Generically, the peaks on both sides of the market differ. Referring to the women as the side with the lowest peak, the second ratio on the right hand side of the assumption is smaller than 1 . Hence, with large enough populations, Assumption A1 is always met.

\subsection{Definitions of Concepts in Networks}

Let $g$ be a network. The elements of $N$ are called vertices. A path in $g$ connecting two vertices $i_{1}$ and $i_{n}$ is a set of distinct nodes (a node is a link between two individuals) in $\left\{i_{1}, i_{2}, \ldots, i_{n}\right\} \subset N$ such that for any $k$, $1 \leq k \leq n-1,\left(i_{k}, i_{k+1}\right) \in g$.

Let $i$ be an individual. We denote by $g(i)=\{j \in N:(i, j) \in g\}$ the set of individuals who have $i$ as a partner in the network $g$. The cardinality of $g(i)$ is called the degree of $i$. If a set $A$ is included either in $M$ or $W$, then the image of $A$ (or the set comprising all the partners of agents in $A$ ) in the network $g$ is denoted by $g(A)=\bigcup_{i \in A} g(i)$.

We denote respectively by $M(g)=\{i \in M: \exists j \in W,(i, j) \in g\}$ and by $W(g)=\{i \in W: \exists j \in M,(i, j) \in g\}$ the set of men and women who are matched in the network $g$. We pose $N(g)=M(g) \cup W(g)$.

A subgraph $g^{\prime} \subset g$ is a component of $g$ if for any $i \in N\left(g^{\prime}\right)$ and $j \in N\left(g^{\prime}\right)$ such that $i \neq j$, there is a path in $g^{\prime}$ connecting $i$ and $j$, and for any $i \in N\left(g^{\prime}\right)$ and $j \in N(g)$ such that $(i, j) \in g,(i, j) \in g^{\prime}$. Intuitively, a component of $g$ is a maximal subset of directly or indirectly connected agents in $g$. A network $g$ can always 
be partitioned into its components. This means that if $C(g)$ is the set of all the components of $g$, then $g=\bigcup_{g^{\prime} \in C(g)} g^{\prime}$, and for any $g^{\prime} \in C(g)$ and $g^{\prime \prime} \in C(g), g^{\prime} \cap g^{\prime \prime}=\emptyset$ (two distinct components share no agents; they are like two "social islands").

\section{Pairwise Stable Networks}

In an economy such as the one we are describing, individuals form new links or sever existing links based on the improvement that the resulting network offers them relative to the current network. We say that a network $g$ is pairwise stable if: $(i)$ no individual has an incentive to sever an existing link he/she is involved in; and ( $i$ i) no pair of a man and a woman have an incentive to form a new link between them while possibly at the same time severing some of the existing links they are involved in.

More formally, given a profile of utility functions $u=\left(u_{i}\right)_{i \in N}$, a network $g$ is pairwise stable with respect to $u$ if:

(i) $\forall i \in N, \forall(i, j) \in g, u_{i}\left(s_{i}(g)\right) \geq u_{i}\left(s_{i}(g \backslash\{(i, j)\})\right)$; and

(ii) $\forall(i, j) \in(M \times W) \backslash g$, if network $g^{\prime}$ is obtained from $g$ by adding the link $(i, j)$ and possibly severing other links involving $i$ or $j, u_{i}\left(s_{i}\left(g^{\prime}\right)\right)>u_{i}\left(s_{i}(g)\right) \Longrightarrow u_{j}\left(s_{j}\left(g^{\prime}\right)\right) \leq u_{j}\left(s_{j}(g)\right)$ and $u_{j}\left(s_{j}\left(g^{\prime}\right)\right)>u_{j}\left(s_{j}\left(g^{\prime}\right)\right) \Longrightarrow$ $u_{i}\left(s_{i}\left(g^{\prime}\right)\right) \leq u_{i}\left(s_{i}(g)\right)$

According to $(i i),(i, j)$ is a blocking pair whenever the two parties involved strictly benefit from the union. In this sense, link formation is driven only by self-interest, and so, an agent does not enter a relationship if he/she has no incentives to do so. ${ }^{12}$ In this, our definition is different from the one introduced by Jackson and Wolinsky (1996), where two agents form a link if one is willing to do so and the other is indifferent.

To illustrate this definition, consider the following examples. A network in which a woman is matched to $s>s_{w}^{*}$ men is not pairwise stable as she can unilaterally sever $s-s_{w}^{*}$ links. A network in which a man is matched to $s_{m}^{*}+2$ women and a woman not matched to him is matched to fewer than $s_{w}^{*}$ men is not stable, as they could form a link while the man could sever three of his former links (alternatively, the man alone could sever only one of his links). Finally, a network in which a man and a woman who are unmatched have fewer than their optimal partners is not pairwise stable either, as they could form a link without severing any other.

\subsection{Characterization of the Pairwise Stable Networks}

In this subsection, under Assumption A1, we characterize the pairwise stable networks. This characterization will be useful in our dynamic analysis later on. It says that a network is pairwise stable if and only if each woman has exactly her optimal number of partners and each man has at most his optimal number of partners.

Theorem 1 Assume A1, and let $g$ be a network. Then, $g$ is pairwise stable if and only if $\forall(m, w) \in M \times W$, $0 \leq s_{m} \leq s_{m}^{*}$ and $s_{w}=s_{w}^{*}$.

\footnotetext{
${ }^{12}$ In the absence of side payments, the strict improvement of each individual in the pair is a natural assumption (see, e.g., Aumann (1959)).
} 
The intuition for the theorem is simple enough. One could view men making offers to women in sequence, who accept offers until they reach their optimum.

Let us illustrate Theorem 1 with the following example.

Example 1 Consider a matching problem in which there are 10 men and 10 women. Assume that their utility functions are such that $s_{w}^{*}=2$ and $s_{m}^{*}=4$. The three networks represented respectively by Figure 1-1, Figure 1-2 and Figure 1-3 are pairwise stable. In fact, in each graph, each woman has 2 partners (the optimal number of partners for each woman), and each man has at most 4 partners. In the first network component configuration $[(2,2) ;(5,5) ;(3,3)]^{13}$, all agents have 2 partners, thus this network is egalitarian; in the second network component configuration $[(7,6) ;(2,4) ;(1,0)]$, 2 men have 1 partner each, 5 men have 2 partners each, 2 men have 4 partners each, and 1 man has no partner; in the third network component configuration $[(2,4) ;(1,0) ;(1,0) ;(2,2) ;(1,0) ;(1,0) ;(2,4)]$, 2 men have 2 partners each, 4 men have 4 partners each, and 4 men have no partner. An interesting feature of the last two graphs is the uneven share of female partners among men, which reveals a sharp competition in the latter group.

\section{A Dynamic Network Formation Process}

In this section, we turn to dynamics. First, we shall define a Markov process for any given matching problem as previously defined, to describe the formation and severance of links over time. Later on, given the lack of predictive power of this process, we shall resort to perturbing it in two different ways, leading to two perturbed Markov processes, studied in Sections 5 and 6, respectively.

The unperturbed Markov process, labelled $P^{0}$, is as follows. Time is discrete. In each period, a man and a woman chosen at random with arbitrary positive probability are given the opportunity to sever or add a link based on the improvement that the resulting network offers to them relative to the current network. If they are already linked in the current network, the decision is whether to sever the link. Otherwise, the decision is whether to form a new link. While forming a new link, each agent is allowed to sever as many of the links he/she is involved in as possible in the current network. Link severance is unilateral, while link formation is bilateral.

Let $g$ and $g^{\prime}$ be two networks. They are said to be adjacent if $g^{\prime}$ is obtained from $g$ by an agent severing an existing link he/she is involved in in $g$, and possibly forming a new link with an agent of the opposite type. More formally, $g$ and $g^{\prime}$ are adjacent if there exist $i \in M$ and $j \in W$ such that $g^{\prime} \in\{g+i j, g+i j-i k, g+i j-$ $i k-j m, g+i j-j m, g-i j\} .{ }^{14}$ Let $x$ and $y$ be two networks. An $(x, y)-$ path is a finite sequence of networks $\left(g^{0}, g^{1}, \ldots, g^{k}\right)$ such that $g^{0}=x, g^{k}=y$, and for any $t \in\{0,1, \ldots, k-1\}, g^{t}$ and $g^{t+1}$ are adjacent.

\footnotetext{
${ }^{13}[(2,2) ;(5,5) ;(3,3)]$ refers to a network component configuration with 3 components, the first containing 2 men and 2 women, the second 5 men and 5 women, and the third containing 3 men and 3 women. This notation is a simplification that abstracts from the complete network structure as represented by the graph.

${ }^{14}$ We simplify notation here and write $i j$ instead of $(i, j), g+i j$ instead of $g \cup\{(i, j)\}$, and $g-i j$ instead of $g \backslash\{(i, j)\}$, etc.
} 
An improving path from $x$ to $y$ is a finite sequence $x=g^{0}, g^{1}, \ldots, g^{k}=y$ such that for any $t \in\{0,1, \ldots, k-$ $1\}$, the transition from $g^{t}$ to $g^{t+1}$ strictly benefits its initiator(s). More formally:

- $(i) g^{t+1}=g^{t}-i j$ for some $i j$ such that $u_{i}\left(s_{i}\left(g^{t+1}\right)\right)>u_{i}\left(s_{i}\left(g^{t}\right)\right)$ or $u_{j}\left(s_{j}\left(g^{t+1}\right)\right)>u_{j}\left(s_{j}\left(g^{t}\right)\right)$; or

- $(i i) g^{t+1} \in\left\{g^{t}+i j, g^{t}+i j-i k, g^{t}+i j-i k-j m, g^{t}+i j-j m\right\}$ for some $i j$ such that $u_{i}\left(s_{i}\left(g^{t+1}\right)\right)>u_{i}\left(s_{i}\left(g^{t}\right)\right)$ and $u_{j}\left(s_{j}\left(g^{t+1}\right)\right)>u_{j}\left(s_{j}\left(g^{t}\right)\right)$. Here, without loss of generality, due to our single-peak assumption, there is no need to allow for an agent severing more than one link when forming a new link.

Recurrent classes of a Markov process are those sets of states such that, if reached, the process cannot get out of them, and which do not contain a smaller set with the same property. We next characterize the recurrent classes or steady states of the unperturbed markov process $P^{0}$.

Theorem 2 The recurrent classes of the unperturbed markov process $P^{0}$ are singletons, whose union coincides with the set of pairwise stable networks.

We view Theorems 1 and 2 as safety checks for the model, showing that it can offer sensible predictions. Thus, the set of long-run predictions of the unperturbed dynamics is quite large (recall the characterization in Theorem 1). We proceed by perturbing this process in the sequel. We shall define below two such perturbed processes. To better frame our contribution in the literature, we shall also use a very simple example to compare the predictions of our two processes to those of another process proposed by Jackson and Watts (2002).

\section{$5 \quad$ The First Perturbed Markov Process $P_{1}^{\varepsilon}$}

In this section, we define and analyze the first perturbed process. In each period, the revision opportunity offered at random to a male-female pair is the same as described in the process $P^{0}$. However, now agents may make decisions that do not necessarily lead to an immediate individual improvement. We describe these events in detail.

- If the two agents are linked in the current network:

- Link severance takes place with probability 1 if it benefits either of the two agents, just as before.

- Otherwise, while in the unperturbed process, no severance of this link was taking place, now if it makes the two agents worse off, severance takes place with probability $\varepsilon$ (note that in our model, link severance cannot make an agent indifferent). Recall that link severance is a unilateral decision, and thus it takes one "mistake" to sever such a good link: an agent making a mistake with probability $\varepsilon>0$.

- If the two agents are not linked in the current network, the decision is whether to form a new link: 
- This link formation takes place with probability 1 if it is mutually beneficial, just as before. All other transitions did not happen in the unperturbed process, while now they will.

- If forming the link makes one agent worse off and the other better off -one "mistake"-, it occurs with probability $\varepsilon$.

- If the link formation makes the two agents worse off -two "mistakes"-, it occurs with probability $\varepsilon^{2}$.

- If the transition makes one agent better off and the other agent, say $j$, indifferent, agent $j$ may take this "neutral action" and looks at considerations other than his/her well-being. Indifference in the transition happens because, while forming a new link with $i, j$ severs an existing link, say with agent $k$ in the current network. Then, the resistance of this transition amounts essentially to the perceived strength (or quality) of the severed link. Specifically, we assume that the transition occurs with probability $\varepsilon^{f\left(\frac{1}{s_{k}}\right)}$ where the link strength $f$ is a strictly increasing function of $\frac{1}{s_{k}}$ mapping into $(0,1)$. Here, $s_{k}$ is the number of partners that $k$ has in the current network. We offer an interpretation below, at the end of the description of the process.

- If the transition makes one agent worse off and the other agent indifferent (one "mistake" and one "neutral action"), the transition occurs with probability $\varepsilon * \varepsilon^{f\left(\frac{1}{s_{k}}\right)}=\varepsilon^{1+f\left(\frac{1}{s_{k}}\right)}$.

- Finally, if it makes the two agents indifferent (two "neutral actions"), meaning that while forming a new link, $i$ and $j$ severed links with, say $h$ and $k$, respectively in the current network, it occurs with probability $\varepsilon^{f\left(\frac{1}{s_{h}}\right)} * \varepsilon^{f\left(\frac{1}{s_{k}}\right)}=\varepsilon^{f\left(\frac{1}{s_{h}}\right)+f\left(\frac{1}{s_{k}}\right)}$.

We emphasize our assumption on the resistance of transitions involving indifferences or "neutral actions", the key transitions for our results. The function $f\left(\frac{1}{s_{k}}\right)$ can be viewed as the perceived strength of the link that is being severed by $j$. If we assume for instance that each agent is endowed with 1 unit of time that he/she splits equally among all his/her partners, then it makes sense to assume that the strength of a link is inversely proportional to the number of partners. ${ }^{15}$

The time invested in a relationship may also be viewed as proof of commitment to the other partner. ${ }^{16}$

\subsection{Resistance of a Path and Stochastic Stability}

For any adjacent networks $g$ and $g^{\prime}$, the resistance of the transition from $g$ to $g^{\prime}$, denoted $r\left(g, g^{\prime}\right)$, is the weighted number of agents directly involved in the transition who do not find this change profitable; it is the

\footnotetext{
${ }^{15}$ Although for simplicity, we assume that $j$ observes $s_{k}$, slightly weaker assumptions would do, as $j$ could evaluate the strength $f\left(\frac{1}{s_{k}}\right)$, for instance through a noisy signal, such as the amount of time spent by the partner out of the house. We do not model incomplete information in this paper: a next step in the analysis of the model would be not to assume observability of the number of your partner's partners. For the use of stochastic stability, the agent may not be aware of the exact probability of each event happening, which is just a parameter of the overall dynamics.

${ }^{16}$ One could consider a related model that avoids perturbations of the basic Markov process. In it, agents' preferences are lexicographic with respect to number of links and neutral actions in that order (that is, partner's number of partners). However, we note that the models are not equivalent. Our stochastic processes always lead to Pareto-efficient networks, whereas networks obtained under lexicographic preferences are not Pareto-efficient in general.
} 
exponent of $\varepsilon$ in the corresponding transition probability. We explicitly define $r\left(g, g^{\prime}\right)$ in the table below, as a function of the possible frictions - "mistakes" or "neutral actions"- found in a randomly chosen pair $(i, j)$. To read the table, note that there are only three actions that either $i$ or $j$ can take, some combinations of which might not be possible:

$A$ - Forming a new link without severing an existing link.

$B$ - Forming a new link while severing an existing link. ${ }^{17}$

$C$ - Severing an existing link.

Let $\left(a_{i}, a_{j}\right)$ be the pair of actions taken by $i$ and $j$, respectively. Then $\left(a_{i}, a_{j}\right) \in\{(A, A),(A, B),(B, B),(C, C)\}$. A pair of actions $\left(a_{i}, a_{j}\right)$ might make either agent better off $(b)$, lose $(l)$, or indifferent $(i)$. Transition probabilities and resistances are summarized in Table 1 below.

\section{Table 1}

$\begin{array}{cccc}\left(a_{i}, a_{j}\right) & \text { Outcomes } & \text { Probability } & r\left(g, g^{\prime}\right)=\log _{\varepsilon} \text { (probability) } \\ (A, A) & (b, b) & 1 & 0 \\ (A, A) & (b, l) & \varepsilon & 1 \\ (A, A) & (l, l) & \varepsilon^{2} & 2 \\ (A, B) & (b, i) & \varepsilon^{f\left(\frac{1}{s_{k}}\right)} & f\left(\frac{1}{s_{k}}\right) \\ (A, B) & (l, i) & \varepsilon^{1+f\left(\frac{1}{s_{k}}\right)} & 1+f\left(\frac{1}{s_{k}}\right) \\ (B, B) & (i, i) & \varepsilon^{f\left(\frac{1}{s_{h}}\right)+f\left(\frac{1}{s_{k}}\right)} & f\left(\frac{1}{s_{h}}\right)+f\left(\frac{1}{s_{k}}\right) \\ (C, C) & (b, b) & 1 & 0 \\ (C, C) & (b, l) & 1 & 0 \\ (C, C) & (l, l) & \varepsilon & 1\end{array}$

The resistance of an $(x, y)$-path $q=\left(g^{0}, g^{1}, \ldots, g^{k}\right)$ is the sum of the resistances of its transitions: $r(q)=$ $\sum_{t=0}^{k-1} r\left(g^{t}, g^{t+1}\right)$.

Let $Z^{0}=\left\{g^{0}, g^{1}, \ldots, g^{l}\right\}$ be the set of absorbing states of the unperturbed process (the pairwise stable networks, in our case). ${ }^{18}$ Consider the complete directed graph with vertex set $Z^{0}$, denoted $\nabla$. The resistance of the edge $\left(g^{i}, g^{j}\right)$ in $\nabla$ is the minimum resistance over all the resistances of the $\left(g^{i}, g^{j}\right)-p a t h s: r\left(g^{i}, g^{j}\right)=$ $\operatorname{minimum}\left\{r(q) \mid q\right.$ is an $\left(g^{i}, g^{j}\right)-$ path $\}$.

Let $g$ be an absorbing state. A $g$-tree is a tree whose vertex set is $Z^{0}$ and such that from any vertex $g^{\prime}$ different from $g$, there is a unique directed path in the tree to $g$. The resistance of a $g$-tree is the sum of the resistances of the edges that compose it. The stochastic potential of $g$, denoted $r(g)$, is the minimum resistance over all the $g$-trees.

The set of stochastically stable networks is the set $\left\{g \mid r(g) \leq r\left(g^{\prime}\right)\right.$ for all $\left.g^{\prime}\right\}$ (Young (1993), Kandori, Mailath and Rob (1993)). Intuitively, this set is the set of states (or networks in our case) that are visited a positive proportion of time in the long run. They are also the networks which are the easiest to transition to.

\footnotetext{
${ }^{17}$ Forming a new link while severing more than one link, if not utility improving, is a transition with strictly higher resistance than the one severing only one link, and hence, it can be safely ignored in the subsequent analysis.

${ }^{18}$ Absorbing states are those in singleton recurrent classes.
} 


\subsection{The Result}

We shall now characterize the set of stochastically stable states (or networks) of the perturbed process $P_{1}^{\varepsilon}$. The following definitions and lemmas, of interest in their own right, are needed.

Let $g$ be a network. We shall say that $g$ is egalitarian if all vertices have the same degree; that is, if all individuals have the same number of partners.

Pose $I(g)=\left\{i \in M: s_{i}(g) \geq s_{j}(g) \forall j \in M\right\}$, i.e., the set of men who are linked to the highest number of women in the network $g$.

Let $J(g)=\left\{i \in M: s_{i}(g) \leq s_{j}(g) \forall j \in M\right\}$, i.e., the set of men who are linked to the lowest number of women in the network $g$.

And call $I^{*}(g)=\left\{i \in M: s_{i}(g) \geq s_{w}^{*}\right\}$, i.e., the set of men who have at least a number of partners no less than the women's optimal number.

It is obvious that, if $g$ is pairwise stable, $I(g), J(g)$ and $I^{*}(g)$ are non-empty. Let $L(g)=\sum_{i \in I^{*}(g)}\left(s_{i}(g)-\right.$ $\left.s_{w}^{*}\right)$.

The following lemma states that, under our Assumption A1, any non-egalitarian pairwise stable network (or network in which agents do not all have the same number of partners) is such that any man in $I(g)$ is matched with more than $s_{w}^{*}$ partners, and any man in $J(g)$ is matched with less than $s_{w}^{*}$ partners.

Lemma 1 Assume A1, and let $g$ be a non-egalitarian pairwise stable network. Then, $\forall(i, j) \in I(g) \times J(g)$, $s_{i}(g)>s_{w}^{*}>s_{j}(g)$ (and therefore, $\left.s_{i}(g) \geq s_{j}(g)+2\right)$.

The following lemma describes a simple way to reach egalitarian networks travelling through pairwise stable networks from any initial pairwise stable network.

Lemma 2 Let $g$ be a pairwise stable network. Then, there exists a finite sequence of pairwise stable networks $\left(g^{0}, g^{1}, \ldots, g^{k}\right)$ such that $g^{0}=g, g^{k}=g^{L(g)}$, and $g^{k}$ is egalitarian.

In addition, any two egalitarian pairwise stable networks are "connected" through a path where at least half of it consists of egalitarian pairwise stable networks. This is shown in the following connectivity lemma:

Lemma 3 Let $g$ and $g^{\prime}$ be two distinct egalitarian pairwise stable networks. Then, there exists a finite sequence of pairwise stable networks $\left(g^{0}, g^{1}, \ldots, g^{2 k}\right)$ such that $g^{0}=g, g^{2 k}=g^{\prime}$, and for any $t$ such that $0 \leq t \leq k, g^{2 t}$ is egalitarian.

We are now ready to state the main result of the section:

Theorem 3 Assume A1. A network is stochastically stable in the perturbed process $P_{1}^{\varepsilon}$ if and only if it is egalitarian and pairwise stable. 
The logic behind the proof of Theorem 3, as well as Theorem 4 below, has no connection with that of a model where lexicographic preferences are assumed, which is why these proofs are somewhat involved. Interestingly, our stochastic process implies that if a network that is not efficient is reached (such a network might be the outcome of a model with lexicographic preferences), there exists a path leading out of that network and towards a Pareto-efficient outcome. The interested reader may find illustrations of the workings of these theorems in Pongou and Serrano (2009, 2013), which provide examples to show how networks that are not stochastically stable transition into stochastically stable ones.

\section{The Second Perturbed Process $P_{2}^{\varepsilon}$}

In this section, we define and analyze the second perturbed process. This process is defined as the first perturbed process in Section 5, the only difference being the definition of the probability of a "neutral action", an action that leaves an agent indifferent. Recall that that probability was based on the strength of the link to be broken to form the new link. Now, the perceived strength or quality of such a link will be inversely proportional to the amount of time invested in it. We describe next more formally the only change in assumptions with respect to the previous perturbed process:

- A person who is indifferent in a particular transition, and in it, breaks an existing link with another person who has $s_{k}$ partners in order to form a new link looks at the perceived strength of the link he/she severs. That strength $f\left(s_{k}\right)$ is strictly increasing in $s_{k}$ and strictly bounded between 0 and 1 .

We offer some interpretations of this process. First, in a fidelity economy, this process might correspond to a situation in which an agent who invests too much time in a relationship is perceived as weak or dominated in that relationship. ${ }^{19}$ Second, the time invested by agent $k$ in a relationship with $i$ might be sending a signal regarding the quality of $i$ as a partner to other agents (other agents might think that $i$ should be of high quality for $k$ to dedicate his/her time to her/him). In this light, an individual who has more partners might be perceived as being of higher quality, allowing him/her to attract even more partners. ${ }^{20}$

\subsection{Resistance of a Path}

All the definitions of resistance provided earlier apply to this section as well. For completeness, for each adjacent transition in the perturbed process $P_{2}^{\varepsilon}$, its probability and resistance are summarized in Table 2 below. It uses the same notation employed in Table 1:

\footnotetext{
${ }^{19}$ As noted in the introduction (footnote 8), a possible justification of this assumption comes from the anthropological literature. However, as noted by Pongou and Serrano (2013), the anthropological literature only offers a post-fact rationalization of male domination, whereas the assumption underlying our neutral actions is more general given its gender neutrality: a partner is more likely to be dumped if he/she is perceived as weak, regardless of gender.

${ }^{20}$ This second interpretation is reminiscent of the idea in (Banerjee (1992)) and Bikhchandani, Hirshleifer, and Welch (1992). That is, as in these studies, a signal is likely to induce the phenomenon known as "herd externality" or "informational cascade", although our models are different, as we do not model informational considerations explicitly.
} 
Table 2

$\begin{array}{cccc}\left(a_{i}, a_{j}\right) & \text { Outcomes } & \text { Probability } & r\left(g, g^{\prime}\right)=\log _{\varepsilon} \text { (probability) } \\ (A, A) & (b, b) & 1 & 0 \\ (A, A) & (b, l) & \varepsilon & 1 \\ (A, A) & (l, l) & \varepsilon^{2} & 2 \\ (A, B) & (b, i) & \varepsilon^{f\left(s_{k}\right)} & f\left(s_{k}\right) \\ (A, B) & (l, i) & \varepsilon^{1+f\left(s_{k}\right)} & 1+f\left(s_{k}\right) \\ (B, B) & (i, i) & \varepsilon^{f\left(s_{h}\right)+f\left(s_{k}\right)} & f\left(s_{h}\right)+f\left(s_{k}\right) \\ (C, C) & (b, b) & 1 & 0 \\ (C, C) & (b, l) & 1 & 1 \\ (C, C) & (l, l) & \varepsilon & \end{array}$

\subsection{The Result}

We shall now characterize the set of stochastically stable states of the perturbed process $P_{2}^{\varepsilon}$. The following definition is needed.

Let $g$ be a network. We say that $g$ is anti-egalitarian if $\left\lfloor\frac{s_{w}^{*}}{s_{m}^{*}}|M|\right\rfloor$ men are matched to $s_{m}^{*}$ women each, at most one man is matched to the remaining women (if there is such a remainder), and all other men have no partner.

To understand this definition, the idea is that all women are matched to a set of men that is as small as possible; hence the name "anti-egalitarian." This resembles a one-sided thin economy with a small number of

active sellers and many buyers. Thus, if $\frac{s_{w}^{*}}{s_{m}^{*}}|M|$ happens to be an integer, each of those men is matched to $s_{m}^{*}$ women and the rest of men are unmatched. Note that if $\frac{s_{w}^{*}}{s_{m}^{*}}|M|$ is not an integer, one can assign the remaining women to only one man and have a pairwise stable network. This is because, if one calls $K$ the integer part of that fraction, the total number of links from the set of men not matched to their optimal number must be less than $s_{m}^{*}$ : otherwise, the number of links coming from the men side would be at least $K s_{m}^{*}+s_{m}^{*}$, but this number is strictly greater than $s_{w}^{*}|M|$, the number of links coming from the women side, and both numbers must always be equal.

Equipped with this definition, we state our next result:

Theorem 4 Assume A1. A network is stochastically stable in the perturbed process $P_{2}^{\varepsilon}$ if and only if it is anti-egalitarian and pairwise stable.

\section{Comparison to a Stochastic Process with Uniform Perturbations}

To better place our contribution in the existing literature, we compare the predictions of our two stochastic processes to those of a perturbed process defined in a pioneering study by Jackson and Watts (2002). As in the current paper, Jackson and Watts (2002) assumes discrete time. However, our models differ in that their model assumes uniform probability for all actions that do not increase the utility of their initiator(s). More precisely, faced with the decision to add or sever a link, "the players involved act myopically, adding the link 
if it makes each at least as well off and one strictly better off, and severing the link if its deletion makes either player better off. After the action is taken, there is some small probability $\varepsilon>0$ that a mutation (or tremble) occurs and the link is deleted if it is present, and added if it is absent." (p. 276-277).

It follows that the two models differ in the probability of taking a neutral action. In their framework, unlike in ours, a neutral action is decomposed into two distinct actions taken in two consecutive periods: agent $i$ first severs a link with agent $k$, which occurs with probability $\varepsilon$, and then forms a new link with agent $j$, which occurs with probability 1 (assuming that the added link is beneficial to $j$ as well).

Within our framework, a neutral action is taken in only one period. Also, given that a neutral action is a less severe friction than an action that actually decreases the utility of its initiator, it is more likely to occur in our model, and its probability is therefore strictly greater than $\varepsilon$ (the probability of a proper mistake). The following example shows how the predictions of our two perturbed processes $P_{1}^{\varepsilon}$ and $P_{2}^{\varepsilon}$ differ from those of a perturbed process with uniform perturbations.

An economy involves two men $m_{1}$ and $m_{2}$ and two women $w_{1}$ and $w_{2}$. Utilities are such that the optimal number of partners is 2 for each man and 1 for each woman. There exist four pairwise stable networks $g_{1}, g_{2}$, $g_{3}$, and $g_{4}$ described as follows:

$g_{1}: m_{1} w_{1}\left(m_{1}\right.$ is matched with $\left.w_{1}\right), m_{2} w_{2} ;$

$g_{2}: m_{2} w_{1}, m_{2} w_{2}$

$g_{3}: m_{1} w_{2}, m_{2} w_{1}$

$g_{4}: m_{1} w_{1}, m_{1} w_{2}$

One notes that it takes a neutral action to go from $g_{1}$ to $g_{2}\left(w_{1}\right.$ breaks with $m_{1}$ and matches with $\left.m_{2}\right)$, another neutral action to go from $g_{2}$ to $g_{3}\left(w_{2}\right.$ breaks with $m_{2}$ and matches with $\left.m_{1}\right)$, another neutral action from $g_{3}$ to $g_{4}\left(w_{1}\right.$ breaks with $m_{2}$ and matches with $\left.m_{1}\right)$, and another neutral action from $g_{4}$ to $g_{1}\left(w_{2}\right.$ breaks with $m_{1}$ and matches with $m_{2}$ ).

It can be easily checked that the stochastic potential of each of these networks is 3 when the probability of non-improving actions (proper mistakes and neutral actions) is uniform, as in Jackson and Watts (2002), implying that the set of stochastically stable networks coincides with the entire set of pairwise stable networks. Under our first stochastic process $P_{1}^{\varepsilon}$, only the networks $g_{1}$ and $g_{3}$ are stochastically stable, whereas under our second stochastic process $P_{2}^{\varepsilon}$, only the networks $g_{2}$ and $g_{4}$ are stochastically stable. It follows that our predictions in general differ from those obtained under uniform perturbations, and serve as a useful refinement. In fact, the example also shows that uniform perturbations sometimes fail to refine the set of pairwise stable networks at all.

Jackson and Watts (2002) also define a perturbed process where simultaneous link formation and severance are possible, and apply it to two-sided markets where agents have strict preferences over individual agents or subsets of agents on the other side. Their framework does not apply to our study. Unlike our model, their model does not allow for indifference across networks for agents who have different sets of partners. Also, 
their focus is primarily on one-to-one and many-to-one matching markets, whereas our framework includes one-to-one, many-to-one and many-to-many markets. Furthermore, our findings are qualitatively different. They find that the set of stochastically stable networks coincides with the core. Within our framework, the set of pairwise stable networks coincides with the core, but the set of stochastically stable networks of each of our two stochastic processes refines the core substantially.

\section{Contagion Asymmetry across Economies and Agents Types}

In this section, we answer the question of which side of the economy is more affected by the spread of a random unanticipated information shock. We also show how the concentration of a shock can vary widely across identical economies depending on the "realization" of stochastically stable networks. To this end, we draw on the theoretical framework proposed in Pongou (2010).

Let $g$ be a network. Assume that an agent $i \in N$ is drawn at random to receive a piece of information $\gamma$ that he/she communicates to his/her partners in $g(i)$, who in turn communicate it to their other partners, and so on. If $i$ is not matched with any agent, the information does not spread. Suppose that with equal probability, $\frac{1}{|N|}$, each agent receives the information (i.e., is infected due to a random event). We define the communication or contagion potential of $g$ as the expected proportion of agents who will receive the information. We also define the difference across types in contagion potential as the difference in the expected proportion of men and women who will receive the information. To formally define these notions, consider that $g$ has $k$ components $g_{1}, \ldots, g_{k}$. Pose $I_{k}=\{1, \ldots, k\}$. To simplify notation, we write $N\left(g_{i}\right)=N_{i}, M\left(g_{i}\right)=M_{i}, W\left(g_{i}\right)=W_{i}$, and $\left|N_{i}\right|=n_{i}$ for $i \in I_{k}$. We associate each component $g_{i}$ with the number $n_{i}$ and its bipartite component vector $\left(\left|M_{i}\right|,\left|W_{i}\right|\right)$, and $g$ with the vector $\left[\left(n_{i}\right)\right]_{i \in I_{k}}$ and its bipartite vector $\left[\left(\left|M_{i}\right|,\left|W_{i}\right|\right)\right]_{i \in I_{k}}$. Also, if $g_{i}$ is an isolated component (a component consisting of one agent), its associated vector is either $(1,0)$ or $(0,1)$.

The contagion potential of $g$, denoted $\mathcal{P}(g)$, and the difference across types in the contagion potential of $g$, denoted $\mathcal{F}(g)$, are derived by Pongou (2010) and are provided in the following definition:

Definition 1 Let $g$ be a k-component network with the corresponding component vector $\left[\left(n_{i}\right)\right]_{i \in I_{k}}$.

(1) The communication or contagion potential of $g$ is defined as

$$
\mathcal{P}(g)=\frac{1}{n^{2}} \sum_{i \in I_{k}} n_{i}^{2}
$$

(2) If $g$ is a bipartite graph with the corresponding component vector $\left[\left(\left|M_{i}\right|,\left|W_{i}\right|\right)\right]_{i \in I_{k}}$, the difference across types (or male-female difference) in the contagion potential of $g$ is defined as

$$
\mathcal{F}(g)=\frac{2}{n^{2}} \sum_{i \in I_{k}}\left(\left|M_{i}\right|^{2}-\left|W_{i}\right|^{2}\right) .
$$

Note that our contagion model assumes that the transmission probability is 1 . This assumption is cor- 
rect if contagion means mechanically communicating a received message or a new idea. However, when the transmission probability per interaction is smaller than 1, our assumption is motivated by the fact that we are studying transmission in "long-run equilibrium" or "long-run stable" networks, which implies that contagionprone interactions are repeated over time, causing the transmission probability to approach 1 . In fact, let us assume that the transmission probability per interaction is $\lambda<1$, and that transmission is independent across interactions. Then the transmission probability after $k$ interactions is $1-(1-\lambda)^{k}$, which effectively goes to 1 as $k$ goes to infinity. This logic is justified in our model. Since our comparative statics is on stochastically stable networks, once a stochastically stable network is reached, the system stays there a very long time, only getting out of it after extremely unlikely events. We also note that our model generalizes to situations in which agents communicate information to their partners' partners directly, such as in a classroom where students interact among themselves in addition to interacting with the instructor.

Consider the following illustrative example of the above definition.

Example 2 Consider the networks given in Example 1 and represented respectively by Figure 1-1, Figure 1-2, Figure 1-3 and Figure 1-4. Call them respectively $g, g^{\prime}, g^{\prime \prime}$ and $g^{\prime \prime \prime}$. The contagion potential of each of these networks is: $\mathcal{P}(g)=\frac{1}{20^{2}}\left(4^{2}+10^{2}+6^{2}\right)=\frac{152}{400}=0.38 ; \mathcal{P}\left(g^{\prime}\right)=0.515 ; \mathcal{P}\left(g^{\prime \prime}\right)=0.2 ;$ and $\mathcal{P}\left(g^{\prime \prime \prime}\right)=1$. In the event of a random information shock in, say $g, \mathcal{P}(g)=0.38$ means that $38 \%$ of the population will end up receiving the information following its diffusion.

The difference across types in the contagion potential of each of these networks is: $\mathcal{F}(g)=\frac{2}{20^{2}}\left[\left(2^{2}-2^{2}\right)+\right.$ $\left.\left(5^{2}-5^{2}\right)+\left(3^{2}-3^{2}\right)\right]=0 ; \mathcal{F}\left(g^{\prime}\right)=0.01 ; \mathcal{F}\left(g^{\prime \prime}\right)=-0.12 ;$ and $\mathcal{F}\left(g^{\prime \prime \prime}\right)=0$. Following the diffusion of a random information shock in these networks, these numbers imply that information prevalence will be: equal for men and women in $g$ and $g^{\prime \prime \prime} ; 1$ percentage point greater among men than women in $g^{\prime}$; and 12 percentage points greater for women than men in $g^{\prime \prime}$.

Note how the contagion potential varies across networks despite the fact that the number of links supplied by women and received by men is the same in all networks. This clearly shows the effect of network structure in the propagation of information. It also shows that information prevalence can vary wide across identical economies, as the equilibrium networks "realized" in those economies might be different (for instance, under the first stochastic process, $g$ might realize in one economy, and $g^{\prime \prime \prime}$ in another economy, leading to $38 \%$ of the agents receiving the information in the first economy, and $100 \%$ in the second). Furthermore, we see that $g$ and $g^{\prime \prime \prime}$ are gender neutral in contagion potential; but in network $g^{\prime}$, men are more affected by the information than women, while in network $g^{\prime \prime}$, it is the opposite.

This example also shows that higher optimal number of partners for men than for women does not necessarily cause the latter to be more affected by the spread of a random information shock than the former, when one considers all pairwise stable networks (in $g^{\prime}$, which is a pairwise stable network in the matching problem defined in Example 1, more men than women are affected by the information despite men having a greater 
optimal number of partners than women). But we next show that in the networks that are visited a positive proportion of time in the long run (under our perturbed processes $P_{1}^{\varepsilon}$ and $P_{2}^{\varepsilon}$ ), the ones we are concerned with in the current paper, information never concentrates more among men than among women.

We state below the main result of this section.

Theorem 5 Assume A1.

(1) For any stochastically stable network $g$ of the perturbed process $P_{1}^{\varepsilon}, \mathcal{F}(g)=0$.

(2) For any stochastically stable network $g$ of the perturbed process $P_{2}^{\varepsilon}, \mathcal{F}(g)<0$.

Theorem 5 is illustrated in the following example.

Example 3 There are 3 men and 3 women; $s_{m}^{*}=3$ and $s_{w}^{*}=1$. Consider the networks $g_{1}, g_{2}$ and $g_{3}$ represented respectively by Figures 2-1, 2-2 and 2-3 and by the following component configurations: $[(1,1),(1,1),(1,1)]$, $[(1,2),(1,0),(1,1)]$ and $[(1,3),(1,0),(1,0)]$.

In $g_{1}$, each man is matched to a woman; in $g_{2}$, man $m_{1}$ is matched with two women, while $m_{2}$ is unmatched and $m_{3}$ is still matched with one woman; in $g_{3}, m_{1}$ is matched with all three women while the other men are unmatched. We note that only the egalitarian pairwise stable network $g_{1}$ is stochastically stable under the process $P_{1}^{\varepsilon}$, while only the anti-egalitarian pairwise stable $g_{3}$ is stochastically stable under the process $P_{2}^{\varepsilon} \cdot{ }^{21}$ In addition, we have $\mathcal{F}\left(g_{1}\right)=0$ and $\mathcal{F}\left(g_{3}\right)=-\frac{1}{3}<0$.

Theorem 5 implies that any initial network $g$, if not stochastically stable under $P_{1}^{\varepsilon}$ or $P_{2}^{\varepsilon}$, will transition to a network $g^{\prime}$ that is stochastically stable, in which information prevalence is at least as high among women as among men, even if in the initial network $g$, the prevalence was higher among men than women (see also Pongou and Serrano (2013) for another illustration). Furthermore, in the case of the second process, which under our assumptions may be viewed as a description of male-dominant societies or markets, women concentrate more information than men. ${ }^{22}$

\section{Some Applications}

Earlier, we noted that our model applies to markets where agents derive utility over the number of partners-the volume of trade-- This section covers the implications and suggests interpretations of our theoretical findings for some of these markets.

Multilateral Exchange between Rich and Poor Countries. Our model might be applied to illustrate the patterns of commercial relationships between rich countries $(M)$ and poor countries $(W)$, where the word "rich" (resp. "poor") is broadly defined to include countries whose per capita income is above (resp. below)

\footnotetext{
${ }^{21}$ If we had $s_{m}^{*}=2$, only $g_{2}$ would be stochastically stable under the process $P_{2}^{\varepsilon}$.

${ }^{22}$ In fact, one can show in general that the set of anti-egalitarian pairwise stable networks includes those networks that maximize the difference across types in contagion potential, although the inclusion is strict.
} 
the median of the income distribution, so that $|M|=|W|$. It is well-known that the international market for certain goods such as military goods is very concentrated, being supplied only by a small number of rich countries, whereas other markets (e.g., the market for manufactured foods) are highly fragmented, as they involve a large number of suppliers. To understand these different trade patterns based on our model, one can plausibly argue that the optimal number of commercial partners is greater for rich countries $\left(s_{m}^{*}\right)$ than for poor countries $\left(s_{w}^{*}\right)$. Our first stochastic process may be viewed as follows: forced to replace one trading partner with another, a country stays away from countries with already many other trading partners, in order to avoid long queues in the delivery of goods. This depicts a very competitive market, and it leads countries on each side to have the same number of trading partners. It might therefore shed light on the functioning and outcomes of the international markets for "nonstrategic" goods such as foods and clothing, which involve a large number of suppliers and purchasers.

Our second stochastic process leads to a concentrated market: all the poor countries are matched to only a few number of rich countries. This process might be describing the behavior of agents in the international market for certain "strategic" goods such as military weapons. If poor "neighboring" countries compete for military leadership in their region, they will all purchase weapons and other military goods from the same suppliers in order to make sure that none of the countries has a superior war technology. An illustration of this behavior is Example 3. Assume that the three men stand for the suppliers and the three women are poor countries that are neighbors. Each supplier desires to sell weapons to all the poor countries, but each of the latter countries can purchase weapons from only one country. It is possible that the initial matching is the monogamous matching $g_{1}$. However, if weapons are differentiated products, countries $w_{2}$ and $w_{3}$ might become fearful of supplier $m_{1}$ offering a different or better product to country $w_{1}$ (even if this perception is wrong), leading them to break their respective commercial relationships with $m_{2}$ and $m_{3}$ to match with $m_{1}$. This will ensure that all the poor countries possess the same war technology in the long run. There exist several other interpretations of the second process for the markets that we cover in the sequel.

Buyer-Seller Market. Our model might be applied to understand the patterns of relationships between sellers $(M)$ and buyers $(W)$ of a good for instances where $|M|=|W|$. It is generally the case that the optimal number of buyers $\left(s_{m}^{*}\right)$ for each seller is greater than the optimal number of sellers $\left(s_{w}^{*}\right)$ a buyer can purchase from. Our first stochastic process depicts a situation where buyers, rightly or wrongly, perceive the number of other customers who purchase from a store as reflecting the amount of extra time that they will spend in that store (this perception might be wrong if they are as many cashiers as the optimal number of buyers in a store, but the number of cashiers might not be known to the buyers prior to them deciding to purchase from a specific store). This belief leads to a uniform market structure, in which each seller ends up with an equal share in the overall market.

Our second process might be interpreted as depicting a situation in which the number of buyers who purchase from a seller signals the quality of the latter. A seller who has more customers is therefore perceived 
as being better than a seller who has less, which allows the former to attract even more customers up to his optimal number. In this case, the result might be viewed as reminiscent of a "herd externality" (Banerjee (1992)) or an "informational cascade" (Bikhchandani, Hirshleifer, and Welch (1992)) among customers, in that, in choosing whom to purchase from, they are influenced by the choices of other buyers. As argued by Banerjee (1992), herd behavior characterizes several choices in real life, including the choice of a restaurant, or the choice of a school. Indeed, in our study, herding leads to a one-sided thin economy, in that all buyers purchase from a small number of sellers.

Two-sided labor and academic markets. Our model might also be applied to understand the patterns of relationships between employers $(M)$ and employees $(W)$ in certain industries where labor contracts stipulate a prima facie duty of loyalty of employees to their employers, therefore making it more costly for the former to work for competing firms. This fidelity requirement implies that the optimal number of workers $\left(s_{m}^{*}\right)$ a firm can hire exceeds the optimal number of firms an individual can work for $\left(s_{w}^{*}\right)$. In certain sectors, including national defence, secret services, intelligence, and research in the military or pharmaceutical industry, this loyalty requirement might be very strong, implying $s_{w}^{*}=1$.

Our stochastic processes might then be interpreted in different ways, depending on the sector. The first process depicts a situation where agents on both sides, rightly or wrongly, perceive the time that their partners invest in their relationship as signaling commitment. If the perception is right, then the stochastic process can be viewed as being utility-driven, when an agent cares about the commitment of such labor relationships. Some other considerations may be at play, though, to explain why severing links with partners who hold more links becomes easier (e.g., better working conditions). In either case, the prediction of the model is that of pairwise stable egalitarian networks in the long run: each worker works for her optimal number of firms, and each firm ends up hiring that same number of workers. This depicts a labor market served by many small employers.

In contrast, the second process depicts a situation where the number of partners that an agent has might be a noisy signal of his/her quality. For example, a firm that has many employees might be wrongly perceived by other individuals as treating its employees better than a firm that has less. Similarly, a faculty who has more students to advise might be wrongly perceived by other students as having more ideas than a faculty who has fewer students. In this case, there will be herding among workers or students, leading to pairwise stable anti-egalitarian networks in the long run. That is, we would see a highly concentrated labor market with few large employers (or few professors advising students, while the rest would have no students).

Our second process might also be interpreted as being driven by utility considerations. Indeed, in an enriched version of our model, even if the quality signal sent by the number of partners that an agent has is wrong at the initial stage of network formation (because all agents on each side of the market are initially identical in terms of the characteristics that the agents on the other side value), it might become true over time. For instance, there are many contexts where an employer's managerial experience and skills increase 
with the number of employees she has had. Therefore, it is possible that employers are initially identical, and that a pairwise stable matching between employers and employees forms in the initial period, with some employers having more employees than others. Over time, employers who initially had more employees will have a better reputation than those who had less, even attracting more employees up to their optimal number. The same applies to the faculty-student market. A faculty who has had more students might over time become more skilled at advising and might have a better experience with finding better jobs for his students than his colleague who had no students, even if both were initially identical. In all of these cases, the prediction of the model is that of pairwise stable anti-egalitarian networks in the long run.

Dating and union patterns across cultures. Our analysis may also be used to shed light on dating and union formation patterns in certain societies. Imagine that women's optimal number of partners is 1 (this corresponds to the official constraint on marriage for women in almost all societies). Then, in the first process, the model predicts a situation of serial monogamy. Theorem 3 shows for this case that only monogamous networks are stable in the long run. This notion of stability, however, does not imply that if the process reaches a monogamous network, it will stay there, since people might still make mistakes or be tempted by other potential partners. Indeed, if a woman moves from her only partner to another one, creating a non-monogamous network, the latter network will transition to another monogamous network which is not necessarily the initial one, and so on. Serial monogamy, known to be more prevalent in Western societies, is associated with high divorce rates (e.g., Schoen and Standish (2001) and Goldstein (1999) document that the divorce rate in the U.S. is above 40\%). In contrast, under the second process, the prediction of the model is polygyny, and then divorce rates may be low. Consider the following example. There are 3 men and 3 women, $s_{w}^{*}=1$ and $s_{m}^{*}=3$. Theorem 4 tells us that the only stochastically stable network (up to permutations) is the one in which the first man is matched to all three women. Assume that the process reaches that network. If a woman leaves the first man to match with another man, then considering that networks evolve following the path of least resistance, it is easy to see that that woman will return to the first man (so, there will be reconciliation and no divorce). The model may be suggesting union formation patterns in regions where polygyny coexists with low divorce rates.

Clearly, the second process better describes male-dominant societies. In such societies, as predicted by the model, men invest less time in their relationships with their wives. ${ }^{23}$ It is also possible, as noted earlier, that the number of partners that an individual has sends a positive signal about his/her quality, which helps to attract other partners. Indeed, quoting George Bernard Shaw, Becker (1974) writes that "the maternal instinct leads a woman to prefer a tenth share in a first rate man to the exclusive possession of a third rate." The interpretations that the time invested in a partner either signals his/her dominant role or his/her quality are both consistent with our second process.

\footnotetext{
${ }^{23}$ For example, based on a survey conducted in Nigeria, John C. Caldwell (1976) wrote that "fewer than one-third of wives normally eat with their husbands or seat together on occasion", which markedly contrasts with what is observed in Western societies.
} 
It is also important to note that monogamous and polygynous networks are only the official marital networks, as in general, women are officially allowed to marry only one husband at a time. In reality, data show that men and women cheat on their partners. When cheating is allowed in our model, one wonders whether the networks that result share similar properties with the official marital networks, which are either monogamous or polygynous. Our findings suggest that they do. Indeed, when cheating is taken into account, according to Theorem 3, the sexual network that forms in a society where only monogamy is legal is a union (in the mathematical sense of the word) of monogamous networks. Similarly, it follows from Theorem 4 that the sexual network that forms in a society where polygyny is legal is a union of polygynous networks. In other words, the rationale that governs the formation of official marital relationships is the same that governs the formation of unofficial relationships in monogamous and polygynous cultures.

Networks, Information, and Infections. Our model may also be used to understand how network configuration affects the spread of information or infections, independently of the distribution of links. To illustrate, consider the networks represented by Figures 1-1 and 1-4, respectively. The number of partners for each agent is the same across these networks. However, these networks have different configurations, with there being 3 components in Figure 1-1 and only 1 in Figure 1-4. It follows, as noted in Example 2, that the spread of a random piece of information will affect $38 \%$ of agents in the former network, and 100\% of agents in the latter. This shows that network configuration has an independent effect on the diffusion of information. An important implication for cross-country differences in the concentration of sexually transmitted infections is that countries might be identical in terms of population size and the profile of preferences over number of sexual partners, but exhibit large differences in infection prevalence. This is because the probability that the networks that realize in identical economies be identical is very small, given the number of equilibria. Similarly, the concentration of information about a new product, idea or technology might vary widely across populations of firms/workers or professors/students, despite the latter having identical distributions of social relationships.

Our model also sheds light on how network configuration may affect long-run gender asymmetry in information concentration. An instance of information may be a new sex technology that can only be learned from a sexual partner, or a sexually transmitted disease. Theorem 5 reveals that information is equally prevalent among men and women in societies where there is more equality between the sexes, but more women than men are informed or infected in male-dominant societies. Applying this to HIV/AIDS, Pongou and Serrano (2013) show that men and women are equally vulnerable in monogamous societies which are better described by our first stochastic process (i.e., relationships are harder to break the lower the number of partners of one's old partner), whereas women are more vulnerable in polygynous societies which are better described by the second process. ${ }^{24}$ It also seems important to note that the two processes might coexist in the same society,

\footnotetext{
${ }^{24}$ Morris and Kretzschmar (1997) also use a network model to study the differential effects of serial monogamy and concurrent partnerships on the spread of HIV/AIDS, but they do not address the question of its gender gap prevalence.
} 
but in different groups or segments of the population. For example, polygyny (which is an outcome of the second process) has been practised among the Mormons in the United States (Becker (1974)), coexisting with monogamy (an outcome of the first process), which is practised by the rest of the country. Also, in developed countries, immigrants coming from polygynous cultures might tend to exhibit the behavioral outcome of the second process, whereas natives might tend to behave according to the first process. In a situation where the two processes coexist, the information will concentrate more among women than among men, especially in those sectors of society better described by the second process.

In our other applications, news about a sale in a particular store spread equally among the buyers and sellers sides in the first process, whereas the word-of-mouth is more prevalent among buyers in the case of a thin market. This logic might also help to understand the patterns of technological diffusion between rich and poor countries. A shock affecting work conditions in a firm extends equally to both sides if the market is composed of many small firms, while the information travels more among the workers when few firms employ all of them. Finally, in the faculty-students application, new ideas spread among students more easily in the model where a few professors are perceived to be of high quality, drawing large numbers of advisees.

\section{Related Theoretical Literature}

Aumann and Myerson (1988) and Jackson and Wolinsky (1996) pioneered the study of endogenous formation of links among agents. Aumann and Myerson (1988) examine a two-stage game. In its first stage, players form bilateral links resulting in a communication and cooperative structure, to which the Myerson value (Myerson (1977)) is applied in the second stage. ${ }^{25}$ Jackson and Wolinsky (1996) introduce a framework for the study of the stability of networks among self-interested individuals. They develop a notion of pairwise stability of networks, and analyze its relationship with efficiency. ${ }^{26}$ They also define the coauthor model, related to ours, in which indirect links have a negative effect (indirect links do not affect our agents' utility).

As for dynamics within two-sided markets, the type of dynamics in which at each period, a pair of individuals can form and sever links goes back to Roth and Vande Vate (1990). More recently, several papers have also studied the dynamics of network formation using the notion of stochastic stability. Some of these papers include Jackson and Watts (2002) and Feri (2007). Although we also use stochastic stability as a "solution concept", our models, their interpretations, and their applications (to contagion in bipartite settings) differ from those in these studies.

Our paper also studies the endogenous formation of links, but there are some significant differences with previous work. First, our notion of pairwise stability allows for simultaneous link formation and severance and therefore differs from pairwise stability à la Jackson and Wolinsky (1996). Second, our focus is confined

\footnotetext{
${ }^{25}$ For extensions and variants, see Dutta, van den Nouweland and Tijs (1996), and Slikker and van den Nouweland (2001a, b). ${ }^{26}$ Other studies on strategic network formation include Dutta and Mutuswami (1997), Bala and Goyal (2000), Watts (2001), Jackson and Watts (2002), Jackson and van den Nouweland (2005), Page, Wooders and Kamat (2005), Dutta, Ghosal and Ray (2005), Bloch and Jackson (2007). For authoritative monographs on networks, see Vega-Redondo (2007), Jackson (2008), and Easley and Kleinberg (2010).
} 
to networks where agents only decide the number of partners they desire, yielding simple characterizations given our assumptions. And third, our dynamic analysis rests on the notion that different transitions in link formation or severance have different probabilities (the different likelihood of our neutral actions), as opposed to uniform mistakes as is customary in the literature.

Another distinctive feature of our model is that we avoid the standard coordination problem by looking at a continuous problem rather than a discrete one. Agents maximize in a continuous way their utility function to determine their optimal number of partners. A similar approach is adopted in Cabrales, Calvó-Armengol and Zenou (2011). ${ }^{27}$ As in this study, agents in our model do not direct their links but decide the number of partners. What is key is the fact that the link formation process is not equivalent to elaborating a nominal list of intended relationships, as is the case in the literature on network formation. Network formation is therefore not the result of an earmarked socialization process, which enables us to totally characterize the pairwise stable matchings, something that has proved rather difficult in the standard framework.

Furthermore, compared to the dynamic network formation literature, our analysis innovates in that individuals do not form links at random as it has often been assumed (see, e.g., the preferential attachment model à la Jackson and Rogers (2007)), but choose links that maximize their myopic utility. Similarly, individuals do not delete links at random but in a strategic way. Indirect links, however, do not matter in our model, as $a$ priori agents may not even know their partners' other partners in certain applications, which enables a clean though not trivial characterization of long-run equilibria.

Our results on pairwise stability also relate to the literature on stability in many-to-many matching markets (e.g., Echenique and Oviedo (2006)). Indeed, if one defines the core with respect to strict coalitional improvements, the core will coincide with our set of pairwise stable networks.

Finally, our paper also connects with the literature on social influence, social learning and contagion (see, e.g., Jackson and Rogers (2007b), Jackson and Yariv (2007), Lopez-Pintado (2008), Young (2009)). The different approaches used in these studies to analyzing diffusion generally assume a connectivity distribution of the population, and/or a payoff function whose arguments include an individual's and her neighbors' choice of a certain behavior, and often rely on mean-field approximation theory to identify equilibria. Each individual faces the choice of adopting a certain behavior, such as buying a new product or not, and this behavior spreads as it is adopted. Our model differs in that it mostly studies "information transmission", not "information adoption." Distinguishing between the two notions is important. Within our framework, an agent who receives information about, say a new product, idea, or technology, communicates it to her friends, but we do not pose the receiver's choice problem. Also, in our application to sexual networks, an agent who is infected with a virus that spreads through sex infects his/her sexual partners; the latter do not make the choice of becoming infected, and the former may not even be aware of his/her status (in this sense, we are closer to the literature on epidemiological contagion (see, e.g., Pastor-Satorras and Vespignani (2000, 2001)).

\footnotetext{
${ }^{27}$ Several other papers have studied link formation based on utility considerations (see, e.g., Snijers (2001), and Staudigl (2011)).
} 
We also note, as remarked by Young (2009), that most papers on social diffusion assume, unlike we do, infinite populations and purely random meetings between individuals.

\section{Conclusion}

We view our contribution as two-fold. First, we have proposed a dynamic model of network formation in two-sided economies. Under our general assumptions, we have characterized static equilibria (pairwise stable networks), as well as long-run equilibria (stochastically stable networks) under two different cultures and perceptions of multiple partnerships. Second, the findings reveal that the configurations of long-run networks are such that the spread of any random unanticipated information would (weakly) affect more women than men. Information concentration might differ across identical economies as well. Furthermore, we have shown that our model can be applied to understand the patterns of relationships in several two-sided markets, including rich/poor countries, buyer-seller, employer-employee, dating, and faculty-student markets. In each of these markets, our stochastic processes potentially have different interpretations, providing a rationale for patterns of fragmentation and concentration that are usually observed.

A distinctive feature of the networks we have studied is that a priori, agents may not know their partners' other partners. In addition, they may not gain anything from these indirect connections. A natural extension of our analysis will be to consider the case in which an individual's well-being is affected by indirect "invisible" links and their consequent externalities. Our basic framework should be amenable to this and other realistic extensions, once incomplete information is incorporated to the analysis.

\section{Proofs}

\section{Proof of Theorem 1.}

Proof. $(1) \Longrightarrow(2)$ : Let $g$ be a pairwise stable network. It is straightforward that $\forall(m, w) \in M * W, 0 \leq$ $s_{m} \leq s_{m}^{*}$ and $0 \leq s_{w} \leq s_{w}^{*}$. In fact, if an agent has more than his/her optimal number of partners, he/she will be better off by unilaterally severing one link, thus implying that $g$ is not pairwise stable, a contradiction.

Therefore, it only remains to show that $\forall w \in W, s_{w}=s_{w}^{*}$. By contradiction, suppose that there exists a woman $w_{0}$ with $s_{w_{0}}<s_{w}^{*}$. First, it should be clear that for every man $m$ not matched with $w_{0}, s_{m}=s_{m}^{*}$. This is because, if at least one such man were matched with fewer women, that man and $w_{0}$ would improve by forming a new link, implying that $g$ is not pairwise stable, which is a contradiction.

It then follows that the number of links coming from the men side is at least $\left(|M|-s_{w_{0}}\right) s_{m}^{*}$, which is greater than or equal to $\left[|M|-\left(s_{w}^{*}-1\right)\right] s_{m}^{*}$, which by Assumption A1 is greater than $|M| s_{w}^{*}=|W| s_{w}^{*}$, an upper bound on the number of links coming from the women side. Since the number of links coming from the men side must exactly equal the number of links coming from the women side, this is impossible. We conclude that $\forall w \in W, s_{w}=s_{w}^{*}$. 
$(2) \Longrightarrow(1)$ : Let $g$ be a network. Assume that $\forall(m, w) \in M * W, 0 \leq s_{m} \leq s_{m}^{*}$ and $s_{w}=s_{w}^{*}$, and let us show that $g$ is pairwise stable. A man alone cannot improve by severing a link since he is at the upward sloping part of his utility function. He cannot form a new link with another woman since each woman has her optimal number of partners. And a woman cannot be part of any blocking move (either by herself or with a man) since she is at her peak. Therefore, $g$ is a pairwise stable network.

\section{Proof of Theorem 2.}

Proof. Let $g$ be a pairwise stable network. No agent can thus be part of a blocking move either by himself/herself or with another agent, implying that there is no improving path leading out of $g$. $\{g\}$ is therefore a recurrent class of $P^{0}$. Conversely, if $g$ is not pairwise stable, it cannot be part of a recurrent class of $P^{0}$. First, it is clear that if $g$ has some agents to the right of their peaks, unilateral severance of links will constitute an improving path out of $g$, leading to strict individual improvements that put every agent weakly to the left of their peaks. But then, if $g$ is not pairwise stable, it must be the case that at least one woman has strictly less partners than at her peak, and since there must be at least one man with the same property, such a link will be formed in an improving path, never to return to $g$. This contradicts that $g$ is part of a recurrent class of $P^{0}$.

Proof of Lemma 1.

Proof. Appealing to the characterization of pairwise stable networks in Theorem 1 and using the definition of egalitarian networks, the proof is easy and left to the reader.

Proof of Lemma 2.

Proof. Let $g$ be a pairwise stable network. Pose $g^{0}=g$. If $g$ is egalitarian, then $\forall i \in M \cup W, s_{i}(g)=s_{w}^{*}$. Thus $L(g)=\sum_{i \in I^{*}(g)}\left(s_{i}(g)-s_{w}^{*}\right)=0$, implying that the sequence searched for is $(g)$. If $g$ is non-egalitarian, then it is obvious that $L(g)>0$ since from Lemma 1, at least one man has more than $s_{w}^{*}$ partners. There exists a pair of men $\left(i_{0}, j_{0}\right) \in I(g) * J(g)$. Again by Lemma 1 , since $s_{i_{0}}(g) \geq s_{j_{0}}(g)+2$, there exists a woman $k_{0}$ such that $\left(i_{0}, k_{0}\right) \in g$ and $\left(j_{0}, k_{0}\right) \notin g$. Sever the link $\left(i_{0}, k_{0}\right)$, and add the link $\left(j_{0}, k_{0}\right)$; call the resulting network $g^{1}$. It is easy to check that $g^{1}$ is pairwise stable and that $L\left(g^{1}\right)=L(g)-1$. Then, either $g^{1}$ is egalitarian and we are done, or not. That is, repeating the same operation $L(g)-1$ more times induces a sequence $\left(g^{1}, \ldots, g^{L(g)}\right)$ of pairwise stable networks. We have $L\left(g^{L(g)}\right)=L(g)-L(g)=0$. Therefore, in the network $g^{L(g)}$, no man has more than $s_{w}^{*}$ partners. But given that each woman has $s_{w}^{*}$ partners in $g^{L(g)}$, that $|M|=|W|$, and that $\sum_{i \in M} s_{i}\left(g^{L(g)}\right)=\sum_{j \in W} s_{j}\left(g^{L(g)}\right)=s_{w}^{*}|W|$, it is necessarily the case that $\forall i \in M, s_{i}\left(g^{L(g)}\right)=s_{w}^{*}$. Thus $g^{L(g)}$ is pairwise stable and egalitarian.

Proof of Lemma 3.

Proof. Let $g$ and $g^{\prime}$ be two distinct egalitarian pairwise stable networks. Pose $g^{0}=g$. Pose $g^{\prime} \backslash g=\{(m, w)$ : $(m, w) \in g^{\prime}$ and $\left.(m, w) \notin g\right\}$. Since $g$ and $g^{\prime}$ are different, $g^{\prime} \backslash g$ is non-empty. Thus, there exists a pair $\left(m_{0}, w_{0}\right)$ such that $\left(m_{0}, w_{0}\right) \in g^{\prime}$ and $\left(m_{0}, w_{0}\right) \notin g$. Since $g$ and $g^{\prime}$ are egalitarian, this implies that there exists a man $m_{0}^{\prime}$ such that $\left(m_{0}^{\prime}, w_{0}\right) \in g$ and $\left(m_{0}^{\prime}, w_{0}\right) \notin g^{\prime}$. (In fact, if we assumed by contradiction that the 
latter statement were wrong, then it would mean that for any pair $\left(m_{0}^{\prime}, w_{0}\right) \in g$, then $\left(m_{0}^{\prime}, w_{0}\right) \in g^{\prime} ;$ and since $\left(m_{0}, w_{0}\right) \in g^{\prime}$ and $\left(m_{0}, w_{0}\right) \notin g$, this would imply that $w_{0}$ has more than $s_{w}^{*}$ in the network $g^{\prime}$, contradicting the fact that $g^{\prime}$ is egalitarian and pairwise stable.)

Then, in $g$, add the link $\left(m_{0}, w_{0}\right)$ and delete the link $\left(m_{0}^{\prime}, w_{0}\right)$ (this is equivalent to woman $w_{0}$ severing her link with $m_{0}^{\prime}$ to form a new link with $m_{0}$ ), and call the resulting network $g^{1}$. In $g^{1}, m_{0}$ and $m_{0}^{\prime}$ have respectively $s_{w}^{*}+1$ and $s_{w}^{*}-1$ partners, and each woman has $s_{w}^{*}$ partners as in $g$. Thus $g^{1}$ is pairwise stable, but it is not egalitarian. Also, note that $g^{1}$ is (one step) closer to $g^{\prime}$ than $g^{0}=g$ (that is, $g^{\prime} \backslash g^{1} \subset g^{\prime} \backslash g$ ).

We now want to construct $g^{2}$. Let $g^{1}\left(m_{0}\right)=\left\{w \in W:\left(m_{0}, w\right) \in g^{1}\right\}$. There exists a woman $w_{0}^{\prime} \in g^{1}\left(m_{0}\right)$ such that $w_{0}^{\prime} \neq w_{0},\left(m_{0}^{\prime}, w_{0}^{\prime}\right) \notin g^{1}$ and $\left(m_{0}, w_{0}^{\prime}\right) \notin g^{\prime}$ (in fact, since $\left|g^{1}\left(m_{0}\right)\right|=s_{w}^{*}+1>1$ and $w_{0} \in g^{1}\left(m_{0}\right)$, there exists $w_{0}^{\prime} \in g^{1}\left(m_{0}\right)$ such that $w_{0}^{\prime} \neq w_{0}$; now, if by contradiction, we assume that for any such $w_{0}^{\prime}$, $\left(m_{0}^{\prime}, w_{0}^{\prime}\right) \in g^{1}$, then it will turn out that $\left|g^{1}\left(m_{0}^{\prime}\right)\right|=s_{w}^{*}$, which is a contradiction since we know from the last paragraph that $m_{0}^{\prime}$ has exactly $s_{w}^{*}-1$ partners in $g^{1}$; finally, if by contradiction, we assume that for any such $w_{0}^{\prime},\left(m_{0}, w_{0}^{\prime}\right) \in g^{\prime}$, then it will turn out that $g^{\prime}\left(m_{0}\right)=g^{1}\left(m_{0}\right)$, implying that $\left|g^{\prime}\left(m_{0}\right)\right|=s_{w}^{*}+1$, thereby contradicting the fact that $g^{\prime}$ is egalitarian). Therefore, sever the link $\left(m_{0}, w_{0}^{\prime}\right)$, add the link $\left(m_{0}^{\prime}, w_{0}^{\prime}\right)$, and call the resulting network $g^{2}$. It is easy to check that in $g^{2}$, each man and each woman has exactly $s_{w}^{*}$ partners. Thus $g^{2}$ is egalitarian and pairwise stable.

We also note that $g^{2}$ is at least 1 step closer to $g^{\prime}$ (in fact, since $\left(m_{0}, w_{0}^{\prime}\right) \notin g^{\prime}$, severing this link in $g^{1}$ does not take us 1 step further from $g^{\prime}$; also, if possible, one can choose $w_{0}^{\prime}$ in such a way that $\left(m_{0}^{\prime}, w_{0}^{\prime}\right) \in g^{\prime}$, and in that case, $g^{2}$ will be 2 steps closer to $g^{\prime}$; if not, $g^{2}$ will be 1 step closer to $\left.g^{\prime}\right)$.

If $g^{2}=g^{\prime}$, we are done; if not, repeat the same operation as previously by replacing $g^{0}$ with $g^{2}$. That will induce $g^{3}$ and $g^{4}$, and will take us at least one step closer to $g^{\prime}$. In general, since $\left|g^{\prime} \backslash g\right|$ is finite, repeating this operation a finite number of times (at most $\left\lceil\frac{\left|g^{\prime} \backslash g\right|}{2}\right\rceil$ times) induces a finite sequence of pairwise stable networks $\left(g^{0}, g^{1}, \ldots, g^{2 k}\right)$ that ends at $g^{2 k}=g^{\prime}$ and satisfying that for any $t$ such that $0 \leq t \leq k, g^{2 t}$ is egalitarian.

\section{Proof of Theorem 3.}

Proof. The proof is divided in two steps, as follows:

Step 1: Let $g$ be a non-egalitarian pairwise stable network. We shall show that $g$ is not stochastically stable. It suffices to show that there exists a network $g^{\prime}$ such that $r\left(g^{\prime}\right)<r(g)$.

Call $T(g)$ the $g$-tree on which the calculation of $r(g)$ is based. There exists a pair of men $\left(i_{0}, j_{0}\right) \in I(g) * J(g)$. Since from Lemma $1, s_{i_{0}}(g) \geq s_{j_{0}}(g)+2$, there exists a woman $k_{0}$ such that $\left(i_{0}, k_{0}\right) \in g$ and $\left(j_{0}, k_{0}\right) \notin g$. Sever the link $\left(i_{0}, k_{0}\right)$, and add the link $\left(j_{0}, k_{0}\right)$, and call the resulting network $g^{1}$.

Consider now the tree $T(g)$. Let $S\left(g^{1}, T(g)\right)$ be the successor of $g^{1}$ in the tree. Now, in $T(g)$, delete the edge $\left(g^{1}, S\left(g^{1}, T(g)\right)\right)$ that leads away from $g^{1}$ and add the edge $\left(g, g^{1}\right)$. This results in a $g^{1}$-tree that we denote by $T\left(g^{1}\right)$.

Since $T\left(g^{1}\right)$ is not necessarily optimal for $g^{1}$, we have $r\left(g^{1}\right) \leq r(g)-r\left(g^{1}, S\left(g^{1}, T(g)\right)\right)+r\left(g, g^{1}\right)$. Because 
$\forall i \in I\left(g^{1}\right), s_{i}(g) \leq s_{i_{0}}(g)$, we have $r\left(g^{1}, S\left(g^{1}, T(g)\right)\right) \geq f\left(\frac{1}{s_{i_{0}}(g)}\right)=r\left(g, g^{1}\right)$. This is because the cheapest way of getting away from $g^{1}$ (which is pairwise stable) is for a pair of a man and a woman to undertake an action that benefits one of them and leaves the other indifferent; such an action is taken with probability at least equal to $\varepsilon^{f\left(\frac{1}{s_{i_{0}}(g)}\right)}$. This implies that $r\left(g^{1}\right) \leq r(g)$.

If $g^{1}$ is egalitarian, then $r\left(g^{1}, S\left(g^{1}, T(g)\right)\right)=f\left(\frac{1}{s_{w}^{*}}\right)>r\left(g, g^{1}\right)$, implying $r\left(g^{1}\right)<r(g)$. If $g^{1}$ is nonegalitarian, repeat the same operation $L(g)-1$ more times. From lemma 2, that will induce a sequence of pairwise stable networks $\left(g^{1}, \ldots, g^{L(g)}\right)$ where $g^{L(g)}$ is an egalitarian network. The induced sequence of $g^{\ell}$-trees, $1 \leq \ell \leq L(g),\left(T\left(g^{1}\right), \ldots, T\left(g^{L(g)}\right)\right)$ will be such that for any $\ell \in\{2, \ldots, L(g)\}, r\left(g^{\ell}\right) \leq r\left(g^{\ell-1}\right)$ with $r\left(g^{L(g)}\right)<r\left(g^{L(g)-1}\right)$. This obviously implies $r\left(g^{L(g)}\right)<r(g)$, and therefore, $g$ is not stochastically stable.

Recall that in any perturbed finite Markov process the set of stochastically stable states is always nonempty. Step 1 has therefore established that the set of stochastically stable networks of the perturbed process $P_{1}^{\varepsilon}$ is a non-empty subset of the set of egalitarian pairwise stable networks.

Step 2: We shall next show that the set of stochastically stable networks of $P_{1}^{\varepsilon}$ coincides with the set of egalitarian pairwise stable networks. It suffices to show that all egalitarian pairwise stable networks have the same stochastic potential.

Let $g$ and $g^{\prime}$ be any two egalitarian pairwise stable networks, and $r(g)$ and $r\left(g^{\prime}\right)$ their respective stochastic potentials. Call $T(g)$ the $g$-tree on which the calculation of $r(g)$ is based. From Lemma 3, we know that there exists a finite sequence of pairwise stable networks $\left(g^{0}, g^{1}, \ldots, g^{2 k}\right)$ such that $g^{0}=g, g^{2 k}=g^{\prime}$, and for any $t$ such that $0 \leq t \leq k, g^{2 t}$ is egalitarian.

Construct $g^{1}$ from $g$ as in the proof of Lemma 3, and consider the $g$-tree $T(g)$. In it, delete the edge $\left(g^{1}, S\left(g^{1}, T(g)\right)\right)$ that leads away from $g^{1}$ and add the edge $\left(g, g^{1}\right)$. This results in a $g^{1}$-tree that we denote by $T\left(g^{1}\right)$. Note that $r\left(g^{1}, S\left(g^{1}, T(g)\right)\right) \geq f\left(\frac{1}{s_{w}^{*}+1}\right)$ and $r\left(g, g^{1}\right)=f\left(\frac{1}{s_{w}^{*}}\right)$.

Next, construct $g^{2}$ from $g^{1}$ as in the proof of Lemma 3, and consider the $g^{1}$-tree $T\left(g^{1}\right)$. In it, delete the edge $\left(g^{2}, S\left(g^{2}, T\left(g^{1}\right)\right)\right)$ and add the edge $\left(g^{1}, g^{2}\right)$. This results in a $g^{2}$-tree that we denote by $T\left(g^{2}\right)$. We have $r\left(g^{2}, S\left(g^{2}, T\left(g^{1}\right)\right)\right)=f\left(\frac{1}{s_{w}^{*}}\right)$ and $r\left(g^{1}, g^{2}\right)=f\left(\frac{1}{s_{w}^{*}+1}\right)$.

Therefore, noting that $T\left(g^{2}\right)$ is not necessarily optimal as a $g^{2}$-tree, we have that $r\left(g^{2}\right) \leq r(g)-r\left(g^{1}, S\left(g^{1}, T(g)\right)\right)+$ $r\left(g, g^{1}\right)-r\left(g^{2}, S\left(g^{2}, T\left(g^{1}\right)\right)\right)+r\left(g^{1}, g^{2}\right)=r(g)-r\left(g^{1}, S\left(g^{1}, T(g)\right)\right)+f\left(\frac{1}{s_{w}^{*}+1}\right) \leq r(g)$ since $r\left(g^{1}, S\left(g^{1}, T(g)\right)\right) \geq$ $f\left(\frac{1}{s_{w}^{*}+1}\right)$. This establishes that $r\left(g^{2}\right) \leq r(g)$, and by symmetry, going back from $g^{2}$ to $g$, that $r(g) \leq r\left(g^{2}\right)$. Therefore, $r(g)=r\left(g^{2}\right)$.

If $g^{\prime}=g^{2}$, then we have shown that $r\left(g^{\prime}\right)=r(g)$. If $g^{\prime} \neq g^{2}$, repeat the same exercise as previously, constructing $g^{\ell}$ from $g^{\ell-1}$ as in Lemma 3, until $g^{\prime}$ is obtained. This induces a sequence of $g^{t}-$ trees $\left(T(g), T\left(g^{1}\right), T\left(g^{2}\right), T\left(g^{3}\right), \ldots, T\left(g^{2 k}\right)=T\left(g^{\prime}\right)\right)$ satisfying that for any $t$ such that $1 \leq t \leq k, r\left(g^{2 t}\right) \leq$ $r\left(g^{2(t-1)}\right)$. This implies $r\left(g^{\prime}\right) \leq r(g)$. By symmetry, going back in the opposite direction, we also have $r(g) \leq r\left(g^{\prime}\right)$, thus implying $r(g)=r\left(g^{\prime}\right)$, which completes the proof.

Proof of Theorem 4. 
Proof. The proof is again organized in two steps, as follows:

Step 1: Let $g$ be a pairwise stable network that is not anti-egalitarian. We shall show that $g$ is not stochastically stable. It suffices to show that there exists a network $g^{\prime}$ such that $r\left(g^{\prime}\right)<r(g)$.

Consider $T(g)$, the $g$-tree on which the calculation of $r(g)$ is based. We claim that, if $g^{\lambda}$ and $g^{\lambda+1}$ are two pairwise stable networks such that for some $m, m^{\prime}, w, g^{\lambda} \backslash g^{\lambda+1}=\{(m, w)\}$ and $g^{\lambda+1} \backslash g^{\lambda}=\left\{\left(m^{\prime}, w\right)\right\}$, the underlying transition does not involve non-pairwise stable networks: if it did, at least one agent directly involved in it would decrease his or her utility, which implies that the resistance of such a transition would exceed 1, whereas the resistance of the direct transition between the two (being adjacent) is strictly less than 1. A simple induction argument shows that this is still true even if two pairwise stable networks are not adjacent (by constructing a path going from one to the other consisting of direct transitions between pairs of adjacent networks).

Therefore, in any transition described in $T(g)$, only pairwise stable networks are visited. By Theorem 1, we know that each pairwise stable network contains exactly the same number of links, i.e., $s_{w}^{*}|W|$. It follows that each transition described in the tree involves a woman $w$ who severs a link with a man $m$ and replaces it with another link with man $m^{\prime}$. Specifically, the pair $\left(m^{\prime}, w\right)$ is offered the opportunity to revise their situation, and as a result, woman $w$ severs $(m, w)$ and gets matched with $m^{\prime}$.

But then, in describing the transition between any two pairwise networks in $T(g)$, one can, without loss of generality, list the transitions that are required going through each individual woman. That is, starting with the woman with the lowest index who has a different set of men to which she is matched in the two networks, one can describe the required severance/creation of links that takes her from her configuration of men in the original network to the one in the final network, and one can proceed like these with each such woman until the full transition is complete.

Consider then the network $g$, and recall it is not anti-egalitarian. We propose the following algorithm. Without loss of generality, label the men so that $s_{m_{1}}(g) \geq s_{m_{2}}(g) \geq \ldots \geq s_{m_{|M|}}(g)$. Let $m$ be the lowest index such that $s_{m}(g)<s_{m}^{*}$. If there exists $w$ who is matched in $g$ to $m^{\prime}>m$, sever the link $\left(m^{\prime}, w\right)$ and replace it with $(m, w)$. Call the resulting network $g^{1}$. We can have two cases. Either $g^{1}$ is anti-egalitarian, or not. If it is, let $g^{\prime}=g^{1}$. If not, repeat the same step. Note how this algorithm always ends after a finite number of steps, say $k$, in a network $g^{\prime}=g^{k}$ that is anti-egalitarian.

Consider the $g$-tree $T(g)$, and without loss of generality (as the first paragraphs of the proof showed), suppose that the transition $g^{\prime}=g^{k} \rightarrow g^{k-1} \rightarrow \ldots \rightarrow g^{1} \rightarrow g^{0}=g$ constitutes a path of directed links in $T(g)$. Change the direction of this path and consider the transition $g=g^{0} \rightarrow g^{1} \rightarrow \ldots \rightarrow g^{k-1} \rightarrow g^{k}=g^{\prime}$. It is obvious that the rest of edges of $T(g)$, along with these new edges (in which the only change introduced is the direction change of previous links in $T(g))$, constitute a $g^{\prime}$-tree, which we call $T\left(g^{\prime}\right)$.

We claim that $r\left(g^{\prime}\right)<r(g)$. Indeed, $r\left(g^{\prime}\right)$ is no greater than the resistance of $T\left(g^{\prime}\right)$, which is equal to $r(g)+\sum_{\alpha=0}^{k-1}\left[r\left(g^{\alpha}, g^{\alpha+1}\right)-r\left(g^{\alpha+1}, g^{\alpha}\right)\right]$. And note that, by construction of the algorithm described, each 
bracketed term is negative. Indeed, in the transition $g^{\alpha} \rightarrow g^{\alpha+1}$, let $m^{\prime}$ be the man who loses a link in favor of man $m$. We know that $s_{m^{\prime}}\left(g^{\alpha}\right)<s_{m}\left(g^{\alpha+1}\right)$, and therefore, $r\left(g^{\alpha}, g^{\alpha+1}\right)=f\left(s_{m^{\prime}}\left(g^{\alpha}\right)\right)<f\left(s_{m}\left(g^{\alpha+1}\right)\right)=$ $r\left(g^{\alpha+1}, g^{\alpha}\right)$.

We have therefore established that, if $g$ is pairwise stable but it is not anti-egalitarian, it is not stochastically stable in the perturbed process $P_{2}^{\varepsilon}$. Given that the set of stochastically stable networks is non-empty, we just proved that this set is a non-empty subset of the set of pairwise stable and anti-egalitarian networks.

Step 2: We shall now prove that the set of stochastically stable networks of $P_{2}^{\varepsilon}$ coincides with the set of pairwise stable and anti-egalitarian networks. It suffices to prove that all of them have the same stochastic potential.

Let $g$ and $g^{\prime}$ be any two such networks. Assume for simplicity that, in each of them, exactly $\frac{s_{w}^{*}}{s_{m}^{*}}|M|$ men are matched with $s_{m}^{*}$ each. Obviously, this must hold for both $g$ and $g^{\prime} .^{28}$

It is easy to see that there must exist $m, m^{\prime} \in M, m \neq m^{\prime}$ and $w, w^{\prime} \in W, w \neq w^{\prime}$ such that $(m, w) \in g \backslash g^{\prime}$ and $\left(m^{\prime}, w^{\prime}\right) \in g^{\prime} \backslash g$. We propose the following algorithm that transforms $g$ into $g^{\prime}$. For each such pair of links, we describe the following steps:

- First, woman $w$ severs her link to man $m$ and gets matched to man $m_{0}$, where $s_{m_{0}}(g)=0$-we know such a man exists in $g$.

- Second, woman $w^{\prime}$ severs her link to man $m^{\prime}$ and gets matched to man $m$.

- And third, woman $w$ severs her link to man $m_{0}$ and gets matched to man $m^{\prime}$.

And to go back, travel the same steps in reverse.

Consider now an optimal $g^{\prime}$-tree, and call it $T\left(g^{\prime}\right)$. In it, focus on the collection of directed edges connecting $g$ to $g^{\prime}$. By arguments similar to those at the beginning of Step 1 of this proof, one can argue that the transition outlined in the previous algorithm must be part of any optimal tree. (We know that transitions in optimal trees do not go through non-pairwise stable networks. In addition, a resistance of $f\left(s_{m}^{*}\right)$ must be paid every time a link with a man matched to his optimal number is broken, and aside from that, a resistance of $f(1)$ that comes from breaking a link with a man who was unmatched in $g$ and remains unmatched in $g^{\prime}$ is the smallest possible positive resistance in this perturbed process.)

Thus, without loss of generality, let the directed path from $g$ to $g^{\prime}$ in $T\left(g^{\prime}\right)$ be the set of transitions outlined. Now, change the direction of the edges in this path, and let that be the only change introduced to the directed edges of $T\left(g^{\prime}\right)$. Observe that the result is a $g$-tree, which we call $T(g)$.

We will now argue that the stochastic potentials of $g$ and $g^{\prime}$ are the same:

\footnotetext{
${ }^{28}$ If, instead, the number $\frac{s_{w}^{*}}{s_{m}^{*}}|M|$ is not an integer, and one man is matched to the remaining women, the argument is the same, but the notation is slightly more complicated. Again, in this case, both $g$ and $g^{\prime}$ have the same structure of having only one man matched to the remaining women.
} 


$$
r(g)=r\left(g^{\prime}\right)+\sum_{\beta=0}^{k-1}\left[r\left(g^{\beta}, g^{\beta+1}\right)-r\left(g^{\beta+1}, g^{\beta}\right)\right]=r\left(g^{\prime}\right) \text { because } \sum_{\beta=0}^{k-1}\left[r\left(g^{\beta}, g^{\beta+1}\right)-r\left(g^{\beta+1}, g^{\beta}\right)\right]=0 . \text { This }
$$

can be easily established, by induction on the number of links that are different between $g$ and $g^{\prime}$.

Indeed, suppose that $g$ and $g^{\prime}$ differ in the smallest possible number of links, which is two, i.e., there exist $m \neq m^{\prime}$ and $w \neq w^{\prime}$ such that $g \backslash g^{\prime}=\{(m, w)\}$ and $g^{\prime} \backslash g=\left\{\left(m^{\prime}, w^{\prime}\right)\right\}$. Consider the transition $g \rightarrow g^{\prime}$ in $T\left(g^{\prime}\right)$. By our previous arguments, such a transition is as follows:

- First, woman $w$ severs her link to man $m$ and gets matched to man $m^{0}$, where $s_{m_{0}}(g)=0$-we know such a man exists in $g$; the resistance of this step is $f\left(s_{m}^{*}\right)$.

- Second, woman $w^{\prime}$ severs her link to man $m^{\prime}$ and gets matched to man $m$; again, the resistance of this step is $f\left(s_{m}^{*}\right)$.

- And third, woman $w$ severs her link to man $m_{0}$ and gets matched to man $m^{\prime}$; the resistance of this step being $f(1)$.

The resistance of the whole transition is thus $2 f\left(s_{m}^{*}\right)+f(1)$. But notice that travelling the same steps backwards takes us back from $g^{\prime}$ to $g$, with exactly the same resistance.

If $g$ and $g^{\prime}$ differ by more links (note this must always be an even number), we use the fact that the path going from $g$ to $g^{\prime}$ and the same path travelled in the opposite direction are "mirror images" of one another. Thus, since the cheapeast transition must always involve establishing links with unmatched men -like $m_{0}$ in the previous paragraph- (because $f(1)$ is the smallest resistance to be added to the $f\left(s_{m}^{*}\right)$ terms, which must be always there), a replication of the argument detailed in the previous paragraph establishes that the total resistance of travelling from $g$ to $g^{\prime}$ is exactly the same as the one travelling backwards on the same path. This completes the proof.

\section{Proof of Theorem 5.}

Proof. Assume A1.

(1) The proof follows from the fact that in any egalitarian pairwise stable network $g$, there is an equal number of men and women in each component of $g$, from which it follows that $\mathcal{F}(g)=0$.

(2) First remark that in any anti-egalitarian pairwise stable network $g$, the number of women exceeds the number of men for all non-isolated components, with strict inequality for some of them. Let us now show that it follows that $\mathcal{F}(g)<0$. Let $\left[\left(\left|M_{i}\right|,\left|W_{i}\right|\right)\right]_{i \in I_{k}}$ be the bipartite component vector of $g$, of which the first $\ell$ components are non-isolated and the remaining $k-\ell$ are isolated (men). It obviously follows that $\sum_{i \in I_{k}}\left|M_{i}\right|=\sum_{i \in I_{\ell}}\left|M_{i}\right|+k-\ell$ and $\sum_{i \in I_{k}}\left|M_{i}\right|=\sum_{i \in I_{\ell}}\left|W_{i}\right|$ (given that no woman is isolated), which in turn implies $\sum_{i \in I_{\ell}}\left(\left|M_{i}\right|-\left|W_{i}\right|\right)=-(k-\ell)<0$. Remark that each non-isolated component vector $\left(\left|M_{i}\right|,\left|W_{i}\right|\right)$ is such that $\left|M_{i}\right|+\left|W_{i}\right|=n_{i} \geq 2$ since it contains at least one man and one woman. Hence, we have the following: 


$$
\begin{array}{rcc}
\mathcal{F}(g) & = & \frac{2}{n^{2}} \sum_{i \in I_{k}}\left(\left|M_{i}\right|^{2}-\left|W_{i}\right|^{2}\right) \\
& = & \frac{2}{n^{2}}\left\{\sum_{i \in I_{\ell}}\left(\left|M_{i}\right|^{2}-\left|W_{i}\right|^{2}\right)+\sum_{\ell+1 \leq i \leq k}\left(\left|M_{i}\right|^{2}-\left|W_{i}\right|^{2}\right)\right. \\
& = & \frac{2}{n^{2}}\left\{\sum_{i \in I_{\ell}}\left(\left|M_{i}\right|-\left|W_{i}\right|\right)\left(\left|M_{i}\right|+\left|W_{i}\right|\right)+\sum_{\ell+1 \leq i \leq k}\left(1^{2}-0^{2}\right)\right. \\
& = & \frac{2}{n^{2}}\left\{\sum_{i \in I_{\ell}}\left(\left|M_{i}\right|-\left|W_{i}\right|\right) n_{i}+k-\ell\right. \\
\leq & \frac{2}{n^{2}}\left\{2 \sum_{i \in I_{\ell}}\left(\left|M_{i}\right|-\left|W_{i}\right|\right)+k-\ell\right\} \\
& < & 0
\end{array}
$$




\section{References}

Aumann, R. (1959): “Acceptable Points in General Cooperative N-Person Games," in

A. W. Tucker and R. D. Luce, Eds. Contributions to the Theory of Games,

Vol. IV. Princeton: Princeton University Press, 1959.

Aumann, R., and R. Myerson (1988): "Endogenous Formation of Links Between Players and Coalitions: An Application of the Shapley Value," in A. Roth, Ed. The Shapley Value, Cambridge University Press, Cambridge, 1988.

Bala, V., and S. Goyal (2000a): "A Non-cooperative Model of Network Formation," Econometrica $68,1181-1230$.

Banerjee, A.V. (1992): "A Simple Model of Herd Behavior," Quarterly Journal of Economics $107,797-817$.

Becker, G.S. (1974): “A theory of Marriage: Part II,” Journal of Political Economy, 82(2), S11-S26.

Bergin J, and B.L. Lipman (1996): "Evolution with state-dependent mutations," Econometrica 64, 943-956.

Bikhchandani, S., D. Hirshleifer, and I. Welch (1992): "A Theory of Fads, Fashion, Custom, and Cultural Change as Informational Cascades," Journal of Political Economy 100, 992-1026

Bloch, F., and M. Jackson (2007): "The Formation of Networks with Transfers Among Players," Journal of Economic Theory 133, 83-110.

Cabrales, A., A. Calvó-Armengol and Y. Zenou (2011): "Social Interactions and Spillovers: Incentives, Segregation and Topology," Games and Economic Behavior 72, 339-360.

Caldwell, J.C. (1976): "Marriage, the Family and Fertility in Sub-Saharan Africa with Special Reference to Research Programmes in Ghana and Nigeria," in S.A. Huzayyin and G. Acsádi, Eds. Family and Marriage in some African and Asiatic Countries, Research Monograph Series no. 6. Cairo: Cairo Demographic Centre.

Caldwell, J.C., P. Caldwell, and I.O. Orubuloye (1992): "The Family and Sexual Networking in Sub-Saharan Africa: Historical Regional Differences and Present-Day Implications," Population Studies 46, 385-410.

Caldwell, P. (1976): "Issues of Marriage and Marital Change: Tropical Africa and the Middle East," in S.A. Huzayyin and G. Acsádi, Eds. Family and Marriage in some African and Asiatic Countries, Research Monograph Series no. 6. Cairo: Cairo Demographic Centre.

Dutta, B., S. Ghosal and D. Ray (2005): "Farsighted Network Formation," Journal of Economic Theory 122, 143-164.

Dutta, B., and S. Mutuswami (1997): "Stable Networks," Journal of Economic Theory 76, 322-344.

Dutta, B., A. van den Nouweland, and S. Tijs (1995): "Link Formation in Cooperative Situations," International Journal of Game Theory 27, 245-256. 
Easley, D. and J. Kleinberg (2010). Networks, Crowds, and Markets: Reasoning About a Highly Connected World. Cambridge University Press.

Echenique, F., and J. Oviedo (2006): "A Theory of Stability in Many-to-Many Matching Markets," Theoretical Economics 1, 233-73.

Feri, F. (2007): "Stochastic stability in networks with decay," Journal of Economic Theory 135, $442-457$.

Foster, D. P., and H. P. Young (1990): "Stochastic Evolutionary Game Dynamics," Theoretical Population Biology 38, 219-232.

Freidlin, M., and A. Wentzell (1984): Random Perturbations of Dynamical Systems, New York: Springer-Verlag.

Gale, D., and L. Shapley (1962): "College Admissions and the Stability of Marriage," American Mathematical Monthly 69, 9-15.

Jackson, M. O. (2008): Social and Economic Networks, Princeton: Princeton University Press.

Jackson, M.O, and B.W. Rogers (2007a): "Meeting Strangers and Friends of Friends: How Random are Social Networks?," American Economic Review 97, 890-915.

Jackson, M. O., and B.W. Rogers (2007b): "Relating Network Structure to Diffusion Properties Through Stochastic Dominance," The B.E. Press Journal of Theoretical Economics 7, 1-13.

Jackson, M.O., and A. van den Nouweland (2005): "Strongly Stable Networks," Games and Economic Behavior 51, 420-444.

Jackson, M.O., and A. Watts (2002): "The Evolution of Social and Economic Networks," Journal of Economic Theory 106, 265-295.

Jackson, M.O., and A. Wolinsky (1996): "A Strategic Model of Economic and Social Networks," Journal of Economic Theory 71, 4474.

Jackson, M.O., and L. Yariv (2007): "Diffusion of Behavior and Equilibrium Properties in Network Games," American Economic Review 42, 92-98.

Kandori, M., G. Mailath, and R. Rob (1993): "Learning, Mutations and Long Run Equilibria in Games," Econometrica 61, 29-56.

Lopez-Pintado, D. (2008): "Contagion in Complex Networks," Games and Economic Behavior $62,573-590$.

Morris, M., M. Kretzschmar (1997): "Concurrent Partnerships and the Spread of HIV," AIDS 11, 641-648.

Myerson, R. (1977): "Graphs and Cooperation in Games," Mathematics of Operations Research 2, 225-229.

Page Jr., F.H., M.H. Wooders, and S. Kamat (2005): "Networks and Farsighted Stability," Journal of Economic Theory 120, 257-269. 
Pastor-Satorras, R., and A. Vespignani (2000): "Epidemic Spreading in Scale-Free Networks," Physical Review Letters 86, 3200-3203.

Pastor-Satorras, R., and A. Vespignani (2001): "Epidemic Dynamics and Endemic States in Complex Networks," Physical Review E 63, 066-117.

Pongou, R. (2010): The Economics of Fidelity in Network Formation, PhD Dissertation, Department of Economics, Brown University (http://gradworks.umi.com/34/30/3430073.html).

Pongou, R., and R. Serrano (2009): "A Dynamic Theory of Fidelity Networks with an Application to the Spread of HIV/AIDS," Working Paper, Brown University.

Pongou, R., and R. Serrano (2013): "Fidelity Networks and Long-Run Trends in HIV/AIDS Gender Gaps," American Economic Review (Papers and Proceedings) 103(3), 298-302.

Quale, G.R. (1992): Families in Context: A World History of Population. Westport, CT: Greenwood. Roth, A.E. (2007): "Repugnance as a Constraint on Markets," Journal of Economic Perspectives 21, $37-58$.

Roth, A., and J.H. Vande Vate (1990): "Random Paths to Stability in Two-Sided Matching," Econometrica 58, 1475-1480

Schoen, R., W. Urton, K. Woodrow, and J. Baj (1985): "Marriage and Divorce in twentieth Century America Cohorts," Demography 22, 101-114.

Slikker, M., and A. van den Nouweland (2001a): Social and Economic Networks in Cooperative Game Theory, Kluwer Academic Publishers.

Slikker, M., and A. van den Nouweland (2001b): "A One-stage Model of Link Formation and Payoff Division," Games Economic Behavior 34, 153-175.

Snijders, T. (2001): "The Statistical Evaluation of Social Network Dynamics," Sociological Methodology 31, 361-395.

Staudigl, M. (2011): "Potential Games in Volatile Environments," Games and Economic Behavior 72, 271-287.

Tertilt, M. (2005): "Polygyny, Fertility, and Savings," Journal of Political Economy 113, 1341-1371

Vega-Redondo, F. (2007): Complex Social Networks, Econometric Society Monograph: Cambridge University Press.

Watts A. (2001): "A Dynamic Model of Network Formation," Games and Economic Behavior 34, $331-341$.

Young, H. P. (1993): "The Evolution of Conventions," Econometrica 61, 57-84.

Young, H. P. (1998): Individual Strategy and Social Structure: an Evolutionary Theory of Institutions, Princeton University Press.

Young, H.P. (2009): "Innovation Diffusion in Heterogeneous Populations: Contagion, Social Influence, and Social Learning," American Economic Review 99 (5), 1899-1924. 
Figure 1-1

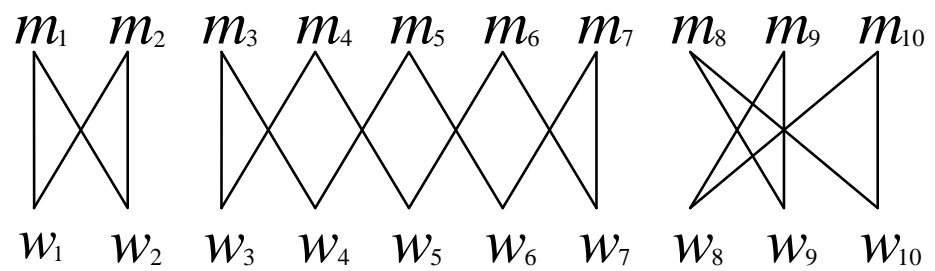

Figure 1-2

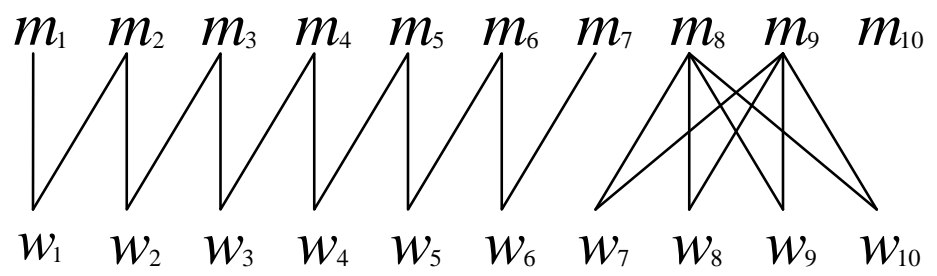

Figure 1-3

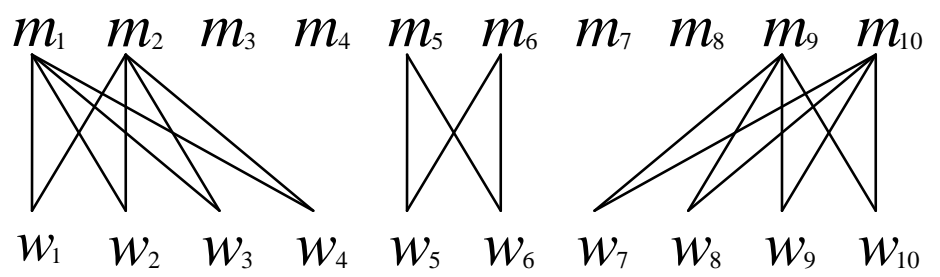

Figure 1-4

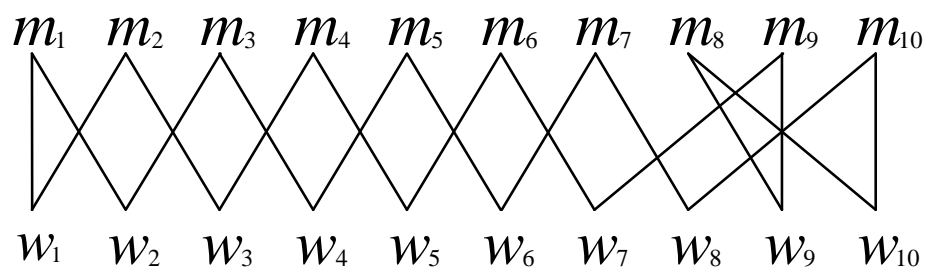


Figure 2-1

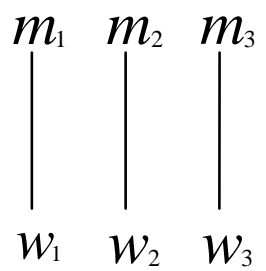

Figure 2-2

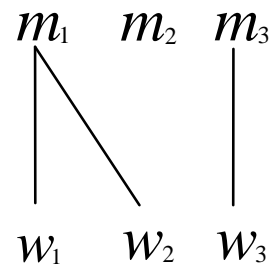

Figure 2-3

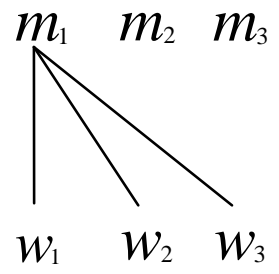

